Comprehensive Summaries of Uppsala Dissertations

from the Faculty of Science and Technology 883

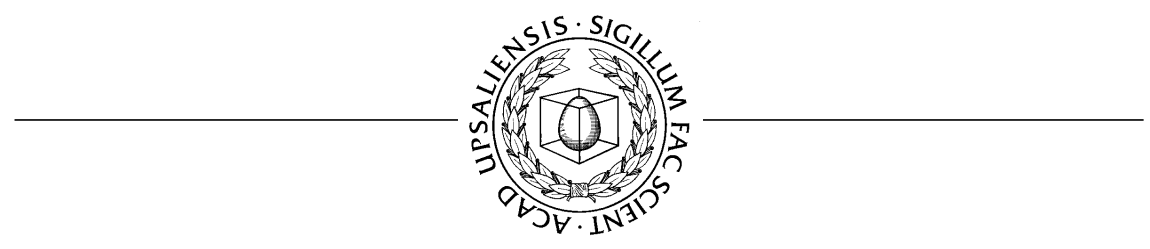

\title{
Searches for the Charged Higgs at Hadron Colliders based on the Tau Lepton Signature
}

BY

YANN COADOU

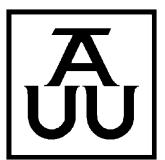

ACTA UNIVERSITATIS UPSALIENSIS

UPPSALA 2003 
Dissertation presented at Uppsala University to be publicly examined in Häggsalen (10132) at the Ångström laboratory, Thursday, October 9, 2003 at 10:15 am, for the degree of Doctor of Philosophy. The examination will be conducted in English.

\section{Abstract}

Coadou, Y. 2003. Searches for the Charged Higgs at Hadron Colliders based on the Tau Lepton Signature. Acta Universitatis Upsaliensis. Comprehensive Summaries of Uppsala Dissertations from the Faculty of Science and Technology 883. 64 pp. Uppsala. ISBN 91-554-5727-4.

The Standard Model of particle physics has been very successful in predicting a wide range of phenomena and has so far been confirmed by all existing data to a very high precision.

The work described in this thesis tests the limits of validity of the Standard Model (SM) in two areas believed to be sensitive to deviations from the theory: the observation of unpredicted particles and CP violation. The studies were performed within the framework of experiments at two hadron colliders, the future ATLAS detector scheduled for operation in 2007 at the Large Hadron Collider in Geneva and the currently running $\mathrm{D} \varnothing$ experiment at the Tevatron in Chicago.

The tau lepton's distinctive signature is a useful tool in many new physics searches where it is present in the final state. As a first study in ATLAS a Monte Carlo analysis of two-tau final states, which are sensitive to the underlying structure of supersymmetric models, was performed.

Several extensions of the SM predict the existence of a charged Higgs boson. The major part of this thesis has consisted in using tau leptons to search for the charged Higgs in the context of the Minimal Supersymmetric extension of the SM (MSSM). Results from this thesis show that searches for the $H^{ \pm} \rightarrow \tau \nu_{\tau}$ decay channel extend the charged Higgs discovery reach for the ATLAS experiment compared to previous studies of other channels: the charged Higgs can be observed for masses up to $\sim 600 \mathrm{GeV}$ for $\tan \beta>10$. Its mass can be determined with an uncertainty of 1 to $2 \%$, dominated by statistical errors. The $\tan \beta$ parameter can be derived from the absolute rate of this decay to a precision around $6 \%$ for $20<\tan \beta<50$.

By measuring precisely the unitarity triangle parameter $\sin 2 \beta$ the SM description of $\mathrm{CP}$ violation can be put to a test. As a separate study a Monte Carlo analysis was performed in ATLAS, which shows that the systematic uncertainty is half the attainable statistical uncertainty.

As part of the effort to search for the charged Higgs in the DØ experiment a trigger algorithm for tau leptons was written, extensively tested and implemented in the experiment. Trigger strategies for events containing taus were designed. These trigger studies will be useful also for many other new physics searches at DØ.

Yann Coadou, Department of Radiation Sciences, Uppsala University, Box 535, SE-751 21 Uppsala, Sweden

(C) Yann Coadou 2003

ISSN 1104-232X

ISBN 91-554-5727-4

Printed in Sweden by Kopieringshuset AB, Uppsala 2003 

This thesis is based on the following papers:

I Identification of Hadronic Tau decays in ATLAS

Y. Coadou, I. Hinchliffe, J. Lozano-Bahilo, L. C. Loveridge and M. D. Shapiro ATLAS Note ATL-PHYS-98-126 (1998)

II Measurement of $\sin 2 \beta$ from $B_{d}^{0} \rightarrow J / \psi K_{s}^{0}$ : statistical reach and estimate of the systematic uncertainties Y. Coadou, J. Damet, H. Korsmo and G. F. Tartarelli ATLAS Note ATL-PHYS-99-022 (1999)

III The Hadronic $\tau$ Decay of a Heavy $H^{ \pm}$in ATLAS

K. A. Assamagan and Y. Coadou Acta Phys.Polon. B33 (2002) 707-720

IV Prospects for the Determination of the Charged Higgs Mass and $\tan \beta$ with the ATLAS Detector at the Large Hadron Collider

K. A. Assamagan and Y. Coadou Acta Phys.Polon. B33 (2002) 1347-1360

$\mathrm{V}$ ATLAS discovery potential for a heavy charged Higgs boson K. A. Assamagan, Y. Coadou and A. Deandrea Eur. Phys. J. direct C4 (2002) 9, hep-ph/0203121

VI The Level 3 Tau Tool

Y. Coadou

DØ Note 4132

Part of the work presented in this thesis has also been published in the following reports:

- Papers I and II in ATLAS Detector and Physics Performance: Technical Design Report, ATLAS collaboration CERN-LHCC-99-014/15 ATLAS-TDR-14/15 (1999)

- Paper II in $B$ decays at the LHC, P. Ball et al. in proceedings of "CERN Workshop On Standard Model Physics (And More) At The LHC", CERN-TH-2000-101, hep-ph/0003238

- Papers III, IV and V in The Higgs working group: Summary report, D. Cavalli et al.

in proceedings of "Les Houches 2001, Physics at TeV colliders" 1-120, hep-ph/0203056 


\section{Contents}

\begin{tabular}{ll}
\hline Introduction & 7
\end{tabular}

1 Theoretical framework $\quad 9$

1.1 The Standard Model . . . . . . . . . . . . . . . . . . . . . . 9 9

1.2 Extensions of the Standard Model . . . . . . . . . . . . . 14

$2 \quad$ ATLAS and the Large Hadron Collider 21

2.1 The next accelerator . . . . . . . . . . . . . . . . . 21

2.2 The ATLAS detector . . . . . . . . . . . . . . . . . . . . 22

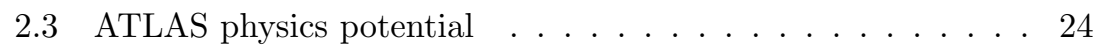

\begin{tabular}{|lll}
3 & Triggering with the $\mathrm{D} \emptyset$ detector & 27
\end{tabular}

3.1 The Tevatron accelerator $\ldots \ldots \ldots$. . . . . . . . . . 27

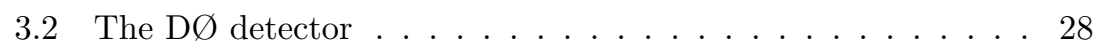

3.3 The $\mathrm{D} \emptyset$ trigger system $\ldots \ldots \ldots \ldots$

\begin{tabular}{|lll}
4 & Summary of papers & 47
\end{tabular}

5 Conclusions and outlook 51

\begin{tabular}{|ll}
\hline Acknowledgements & 53
\end{tabular}

References

\begin{tabular}{|ll|}
\hline A Abbreviations and acronyms & 63
\end{tabular} 



\section{Introduction}

In trying to reveal the unknown microstructures of our Universe, physicists have formulated a theoretical framework which describes the interactions between the elementary constituents of Nature: the Standard Model. It describes in detail what particles should be observable, how they are created and decay and what many of their properties should be.

To show the validity of this theoretical model, or to refute it, huge particle accelerators with the ability to probe the properties of matter have been built around the world. By making precise measurements using complex particle detectors it is possible to check the predictions of the theory.

The present thesis describes some investigations made in this field of research. The first chapter summarises the basic characteristics of the Standard Model, in particular some of its features that can be experimentally tested (CP violation, the origin of mass). Planing ahead, extensions of the Standard Model have been formulated and are introduced: if the Standard Model is not a complete description of our world, other solutions are needed, which ought to be tested as well. A particle of the Standard Model with interesting properties, the tau lepton, is presented. It plays an important rôle as a signature for new physics in the work reported in this thesis.

Chapter 2 describes one of the detectors to be built at the new accelerator at the European Laboratory for Particle Physics (CERN) in Geneva: the ATLAS detector at the Large Hadron Collider. The studies reported in Papers $\mathrm{I}-\mathrm{V}$ have been performed for investigations to be made with this detector. The precision to which CP violation can be measured is reported. The capacity of the detector to observe tau leptons was studied. Detecting a particle that is predicted only by extensions of the Standard Model would be the irrefutable proof of the limits of the Standard Model. The main focus of this thesis has been the possibility to observe such a particle, the charged Higgs boson, with the aid of the tau lepton signature.

Until the startup of LHC, the Tevatron collider at Fermilab in Chicago is the highest energy accelerator in the world at which unexpected discoveries could be made. The D $\varnothing$ experiment at this collider is presented in Chapter 3 together with what has been done within the framework of this thesis to increase its capacity to trigger on tau leptons. Paper VI represents part of this work. 
Chapter 4 contains a summary of each Paper included in the thesis and Chapter 5 presents the conclusions of this thesis and an outlook on the possible future use of tau leptons to search for the charged Higgs boson. 


\section{Chapter 1}

\section{Theoretical framework}

The Standard Model (SM) of particle physics is one of the most successful theories in modern physics. It describes matter in terms of its fundamental constituents and their interactions. The concept of fundamental particle has evolved over time. The particles nowadays considered as elementary are divided into three categories described in Section 1.1.1. Two other important aspects of the theory which are of special relevance for this thesis, namely the Higgs mechanism and CP violation, are introduced in Sections [1.1.2 and 1.1 .3 .

One particle has a special place in this thesis, which is the tau lepton. In Section 1.1.4 is given an overview of the properties of this particle as well as the reasons why the tau lepton is of special interest in particle physics.

For reasons given in Section 1.2, the Standard Model is not regarded as the definite answer to the fundamental questions posed in particle physics. Many extensions of the SM exist, like Supersymmetry, Extra Dimensions and others. The main subject of this thesis is to probe Nature in order to see whether it deviates from the predictions of the SM, e.g. with regard to $\mathrm{CP}$ violation, or if new particles, which do not exist in the SM, can be detected. One such candidate is the charged Higgs boson predicted by Supersymmetry.

\subsection{The Standard Model}

\subsubsection{Particles and interactions}

Matter as we know it is, according to the Standard Model and corroborated by measurements, built up from three types of particles: leptons and quarks, which are all fermions (their internal angular momentum, or spin, is $\frac{1}{2}$ ), and gauge bosons (which have integer spin) [1].

There are six leptons (see Table 1.1): the electron, muon and tau lepton and their associated neutrinos. They are ordered in three families. The 
second and the third families constitute replicas of the first family (electron and electron neutrino), with increasing masses. The charged leptons have one unit of electric charge and a finite mass, while the neutrinos are neutral and considered massless in the simplest version of the SM, although recent results show that they actually have a tiny mass [2].

There are also six quarks, ordered in three families similarly to the leptons (see Table 1.1). This resemblance played an important rôle in the history of the discovery of the elementary particles. The revelation of a new particle in the second or third family triggered new theoretical work to incorporate the new particles in a common scheme, leading to the prediction of the existence of even more particles, which have now all been experimentally observed [3]. Quarks of the top row have a fractional electric charge $+\frac{2}{3}$, while bottom row quarks have a charge $-\frac{1}{3}$. They all carry another kind of charge called colour, which comes in three variants: green, blue and red.

\begin{tabular}{|c|c|c|c|c|}
\hline & \multicolumn{3}{|c|}{ Generation } & \multirow{2}{*}{$\begin{array}{l}\text { Electric } \\
\text { charge }\end{array}$} \\
\hline & I & II & III & \\
\hline \multirow[b]{2}{*}{ leptons } & $e$ electron & $\mu$ muon & $\tau$ tau & -1 \\
\hline & $\begin{array}{l}\nu_{e} \text { electron } \\
\text { neutrino }\end{array}$ & $\nu_{\mu} \begin{array}{l}\text { muon } \\
\text { neutrino }\end{array}$ & $\nu_{\tau}$ tau neutrino & 0 \\
\hline \multirow{2}{*}{ quarks } & $u$ up & $c$ charm & $t$ top & $+2 / 3$ \\
\hline & $d$ down & $s$ strange & $b$ bottom/beauty & $-1 / 3$ \\
\hline
\end{tabular}

Table 1.1: The three generations of fermions.

Unlike leptons, quarks are not observable as free particles. They combine in hadrons: three quarks together form a baryon, while a quark and an antiquark make up a meson (all particles — whether elementary or not have an associated antiparticle: it has the same mass but opposite electric charge).

Leptons and quarks make up all the matter as we know it in the Universe. In this discussion we exclude the recently discovered existence of so-called Dark Matter 4. In order to achieve a complete description, more than just the constituent particles is required: a description of their interactions is also necessary. The Standard Model is a quantum field theory where all constituents are described as fields. The interactions between fermions are described as exchanges of mediating particles, the gauge bosons, associated to the interaction fields.

There are four different forces in Nature, corresponding to the exchange of four types of particles: the electromagnetic force (mediated by the exchange of massless photons) affecting all charged particles, the weak force (mediated by the massive weak bosons $W^{ \pm}$and $Z^{0}$ ) responsible for many nuclear reactions like $\beta$ decay, the strong force (mediated by eight massless gluons carrying colour) binding the quarks together, and the gravitational 
force. This last force is enormously much weaker than the other three and therefore negligible in particle physics. It is not described by the Standard Model.

The Standard Model is a gauge theory built on the $\mathrm{SU}(3) \times \mathrm{SU}(2) \times \mathrm{U}(1)$ symmetry of the three gauge groups describing the three forces: $\mathrm{SU}(3)$ accounts for Quantum Chromodynamics (QCD), which describes the strong interaction, and $\mathrm{SU}(2) \times \mathrm{U}(1)$ corresponds to the electroweak interaction that unifies the weak interaction and electromagnetism, described by Quantum Electrodynamics (QED).

The Standard Model being a gauge theory implies that its Lagrangian is invariant under a certain type of symmetry transformations, which constrains the kind of interactions allowed by the model. A theory also has to be renormalisable to have predictive power. Calculations within the framework of the SM often give rise to divergences which have to be regularised [5]. These two features, gauge invariance and renormalisability, are at the origin of much of the theoretical progress achieved in particle physics. They lead, among other things, to the introduction of the Higgs mechanism described in the next section, and also motivated the development of extensions to the SM as mentioned in Section 1.2 .

\subsubsection{The Higgs mechanism}

The Standard Model, as described so far, has one big flaw: the requirement of gauge invariance forbids the presence of mass terms in the Lagrangian. This means that all gauge bosons are massless by construction, whereas experiments have shown some of them to be heavy objects. The key to this problem is the Higgs mechanism [ 6 , a spontaneous symmetry breaking which occurs when the Lagrangian of a system is invariant under a symmetry group, but the vacuum state is not. Its introduction in the electroweak theory generates the masses of the $W^{ \pm}$and $Z^{0}$ bosons [7].

The technical mechanism consists in introducing an $\mathrm{SU}(2)$ doublet of complex scalars, the Higgs field, which couples to the massless gauge fields through a covariant derivative. This extra field respects gauge invariance and is assumed to have a non-zero vacuum expectation value (vev). Expanding this field around its vev, the Lagrangian can be rewritten in such a way that it exhibits mass terms for the gauge bosons. It also contains interaction terms between the gauge fields and the Higgs field, as well as a mass term for the Higgs field itself [8].

The presence of the Higgs field generates masses for the fermions through the introduction of Yukawa couplings in Higgs-fermions vertices with a strength proportional to their mass. The photon and gluons have no coupling to the Higgs field and remain massless.

The Higgs mechanism predicts the existence of another particle associated to the introduced Higgs field, the Higgs boson. All its properties but its mass are known from the theory, but it has so far eluded experimental 
detection 9. There are good reasons to believe that, if it exists, the Higgs boson will be discovered at the experiments installed at the Large Hadron Collider after 2007 (see Chapter 2). With some probability it might be discovered before that at the Tevatron at Fermilab (see Chapter 3).

\subsubsection{CP violation}

The couplings of the Higgs field to the fermions generate the fermion masses. These couplings also induce a misalignment of the quark mass eigenstates with respect to the eigenstates of the electroweak charges. This mixing allows fermions of heavier families to decay into members of a lighter generation through the weak interaction. By convention, the mixing in the quark sector occurs among the down type quarks (see Table 1.1) and is described by the Cabibbo-Kobayashi-Maskawa (CKM) matrix [10], connecting the electroweak eigenstates $\left(d^{\prime} ; s^{\prime} ; b^{\prime}\right)$ of the down, strange and bottom quarks with their mass eigenstates $(d ; s ; b)$ through a unitary transformation:

$$
\left(\begin{array}{c}
d^{\prime} \\
s^{\prime} \\
b^{\prime}
\end{array}\right)=\left(\begin{array}{lll}
V_{u d} & V_{u s} & V_{u b} \\
V_{c d} & V_{c s} & V_{c b} \\
V_{t d} & V_{t s} & V_{t b}
\end{array}\right) \cdot\left(\begin{array}{c}
d \\
s \\
b
\end{array}\right)=V_{C K M} \cdot\left(\begin{array}{c}
d \\
s \\
b
\end{array}\right) .
$$

In the case of three generations of quarks, as expected in the SM, three generalised Cabibbo-type angles and a single complex phase are needed in order to parameterise the matrix. The Wolfenstein parameterisation [1], which corresponds to a phenomenological expansion in powers of the small quantity $\lambda=\left|V_{u s}\right|=\sin \theta_{c} \approx 0.22$ reads:

$$
V_{C K M}=\left(\begin{array}{ccc}
1-\frac{1}{2} \lambda^{2} & \lambda & A \lambda^{3}(\rho-i \eta) \\
-\lambda & 1-\frac{1}{2} \lambda^{2} & A \lambda^{2} \\
A \lambda^{3}(1-\rho-i \eta) & -A \lambda^{2} & 1
\end{array}\right)+\mathcal{O}\left(\lambda^{4}\right)
$$

Now, the $W$ bosons will couple to $u$-type quarks and a linear combination of $d$-type quarks. As some couplings in the Lagrangian will depend on the complex phase, some states will not transform into their hermitian conjugate under CP transformation, the combination of charge conjugation $\mathrm{C}$ (which replaces all particles by their antiparticles) and parity conjugation $\mathrm{P}$ (which inverts all spatial coordinates). This leads to $\mathrm{CP}$ violation, and its only source in the SM is this complex phase of the CKM matrix.

$\mathrm{CP}$ violation has been observed in the $K$ meson system [12] and more recently in $B$ meson decays by different experiments. Results agree so far with the SM predictions [13].

In order to test the description of CP violation by the SM, the unitarity of the CKM matrix is used. It leads to sets of relations between the coefficients of the matrix, which can be checked experimentally. Such a relation exists for the $B_{d}$ meson system, of interest in this thesis:

$$
V_{u d} V_{u b}^{*}+V_{c d} V_{c b}^{*}+V_{t d} V_{t b}^{*}=0 .
$$


As all such relations (there exit similar ones for the $D, K$ and $B_{s}$ systems), it can be represented by a so-called unitarity triangle in a complex plane with $\rho$ and $\eta$ axes (Wolfenstein parameters). The great interest in the relation of the $B_{d}$ system is justified by the fact that all three sides of the triangle are of comparable amplitude, so that experimental tests are more decisive. Measuring independently the sides and the angles $(\alpha, \beta$ and $\gamma)$, one can overconstrain the triangle, hence testing the validity of the Standard Model.

The precise measurement of the angle $\beta$ from the $B_{d}^{0} \rightarrow J / \psi K_{s}^{0}$ decay is studied in Paper II. The direct decay is not CP-violating, but the $B$ meson oscillation mechanism involves a complex coupling between the top and down quarks. CP violation appears in the interference between the direct decay and the decay after oscillation, and can be measured from the difference in decay rates of the $B_{d}^{0}$ and the $\bar{B}_{d}^{0}$ into the same final state.

\subsubsection{The tau lepton}

As mentioned in Section 1.1.1, there are three charged leptons: the electron, the muon and the tau. Only the electron, being the lightest of the three, is stable. The muon decays to an electron and two neutrinos but its comparatively long lifetime of $2.2 \mu$ s gives it plenty of time to fly through particle detectors in most experiments (in particular those described in chapters 2 and (3) before decaying. In all practical respects the muon behaves as a stable particle in such experiments.

This is not the case for the $\tau$ lepton, with a mean life of $2.9 \cdot 10^{-13} \mathrm{~s}$, which will nearly always decay before it reaches the innermost particle detector layer in an experiment. The presence of the $\tau$ lepton can therefore in general only be inferred from the detection of its decay products. The major decay channels and their respective branching fractions are given in Table 1.2 . The life time of the $\tau$ lepton is five times smaller than that of a $B$ hadron (containing a $b$ quark) and its mass of $1.777 \mathrm{GeV}$ is three times smaller. In $85 \%$ of the decays there is only one charged decay product. These are the reasons why, unfortunately, displaced secondary vertex detection for tau identification is more difficult to implement than for $b$-jet identification and, therefore, is not commonly used.

As can be seen from Table 1.2 taus decay hadronically about $65 \%$ of the time, and will appear in a detector as jets with specific features. The track multiplicity will be low, mostly one and three tracks (the so-called one-prong and three-prong channels). The jet will have a low invariant mass and be narrower than an average QCD jet. These are the basic features used for $\tau$ identification.

What makes $\tau$ leptons interesting in our context is that they can be used as a sensitive probe to detect deviations from the Standard Model predictions. For example the LEP experiments have used the tau as a tool to measure precisely many observables related to the $W$ and $Z$ bosons: 


\begin{tabular}{llc}
\hline \hline & Decay mode & Branching fraction \\
\hline \multirow{4}{*}{ one-prong } & $e \nu_{e} \nu_{\tau}$ & $17.84 \%$ \\
& $\mu \nu_{\mu} \nu_{\tau}$ & $17.37 \%$ \\
& $\pi^{ \pm} \nu_{\tau}$ & $11.06 \%$ \\
& $\pi^{ \pm} \nu_{\tau}+n \pi^{0}$ & $36.91 \%$ \\
\hline \multirow{2}{*}{ three-prong } & $\pi^{ \pm} \pi^{ \pm} \pi^{\mp} \nu_{\tau}$ & $10.01 \%$ \\
& $\pi^{ \pm} \pi^{ \pm} \pi^{\mp} \nu_{\tau}+n \pi^{0}$ & $5.18 \%$ \\
\hline \hline
\end{tabular}

Table 1.2: Tau lepton major decay modes and their branching fractions (from 14]).

polarisation, lepton universality, the lineshape at and above the $Z$ resonance and branching fractions 15. No divergence from the SM predictions has been observed so far.

Taus are also very useful in the search for new physics. Owing to their large mass, taus are often present in the decay of new particles, whose couplings to their decay products are proportional to the final state particle masses. For instance with an extended Higgs sector like in, e.g. Supersymmetry, possible decays to $\tau$ final states are $A / H \rightarrow \tau \tau$ and $H^{ \pm} \rightarrow \tau \nu_{\tau}$. The latter process is the main subject of the studies in Papers III, IV and V. In many supersymmetric models, staus (supersymmetric partners of taus) are produced from squark and gluino decays, and produce taus when they decay. The reconstruction of $\tau$ leptons resulting from the decays of supersymmetric particles will be a crucial tool in the investigation of the underlying supersymmetric structure. The extraction of information from measurements of such taus has been investigated in Paper I. In preparation for new physics searches with the $\mathrm{D} \varnothing$ experiment, studies were made of the use of tau signatures in the trigger. These studies are reported in Paper VI and Chapter 3 .

\subsection{Extensions of the Standard Model}

Despite the impressive successes of the Standard Model both in making valid predictions and in explaining all currently available experimental observations to a very high degree of precision, most physicists believe the SM to be only a good approximation, up to a certain energy scale, of some underlying theory. Beyond this scale a more general theory would be needed, of which the SM is the low energy approximation somewhat similarly to that special relativity is approximated by Newtonian mechanics when velocities are small compared to the speed of light.

Among the different reasons for dissatisfaction with the SM [16] one can cite the following:

- there are too many free parameters (at least 19 of them: three gauge coupling constants, a CP-violating strong interaction parameter, six 
quark masses and three lepton masses, the four parameters of the CKM matrix, the Weinberg angle $\theta_{W}$ and the Higgs boson mass).

- there is no explanation for the different quantum numbers.

- nothing justifies the existence of three families of fermions.

- the origin of mass is not yet clarified (although the SM incorporates a mechanism to produce particle masses).

- known masses are very small ( $100 \mathrm{GeV}$ or less) compared to the other known mass scale, the Planck mass $\left(10^{19} \mathrm{GeV}\right)$ — the hierarchy problem.

- gravity is not included.

In order to find possible solutions to these puzzles, many models and ideas have been proposed. Some of them have already been ruled out because they failed to reproduce experimental data. Other ideas are still around, waiting for confirmation from experiment or for more data to constrain their framework.

Since the Higgs boson has still not been observed, models with extended Higgs sectors are also looked at as there is no formal reason to expect only one Higgs doublet in the theory. Such an extension - predicting the existence of several Higgs bosons, two of which are charged - is described below. Other theories try to address deeper questions such as the hierarchy problem. Among those, supersymmetric theories are very popular. The simplest supersymmetric extension of the SM is discussed in Section 1.2.2.

\subsubsection{Two Higgs Doublet Models}

In the Standard Model the Higgs sector consists of one complex doublet field (see Section 1.1.2). This is the simplest choice to generate the masses of the weak gauge bosons. But one could add more Higgs multiplets, as long as they still satisfy known constraints of the SM. In particular the parameter $\rho=m_{W}^{2} /\left(m_{Z}^{2} \cos ^{2} \theta_{W}\right)$ should be very close to 1 and flavour changing neutral currents (FCNC) should be highly suppressed [8]; in the $\mathrm{SM}, \rho=1$ and there are no FCNC at tree level.

It can be shown that models with Higgs singlets or doublets also satisfy $\rho=1$ [8], while this is in general not the case for other models. This is taken as a good reason for considering only models with singlet and doublet fields.

In extended-Higgs-sector theories, FCNCs, i.e. couplings between a neutral boson and two fermions with different flavours, are usually possible through the mediation of a neutral Higgs field. But if one considers only models with more than one Higgs doublet, it was shown [17 that one can naturally remove FCNCs by requiring that all fermions of a given electric charge couple to no more than one Higgs doublet. 
All these conditions are conveniently combined in the minimal extension of the Higgs sector, the two Higgs doublet models (2HDM), where the Higgs sector consists of two complex scalar doublets. In the 2HDM of type I, one of the Higgs doublets couples only to fermions, while the other couples only to bosons. In the type II $2 \mathrm{HDM}$, one doublet couples only to down-type quarks and leptons and the other to up-type fermions. Because the type II model is required by Supersymmetry (see Section 1.2.2), only this type will be further considered.

Two complex Higgs doublets correspond to eight degrees of freedom. Each doublet has a vacuum expectation value, and their ratio is called $\tan \beta$. Through the Higgs mechanism, three degrees of freedom are used to generate the masses of the electroweak gauge bosons. This leaves five physical states: three neutral Higgs bosons (two CP-even $h, H$ and one CP-odd $A$ ), and a pair of charged Higgs bosons $H^{ \pm}$to be discussed in what follows.

\section{Charged Higgs boson production and decays}

The detection of a charged Higgs boson $H^{ \pm}$would be a distinctive signal of physics beyond the Standard Model since such a particle (a charged spin-0 boson) does not exist in the SM.

Searches for the charged Higgs boson have been carried out at LEP, where the main production mechanism is $e^{+} e^{-} \rightarrow H^{+} H^{-}$, leading to a lower limit of $78.6 \mathrm{GeV}$ for the charged Higgs mass independent of the $H^{ \pm} \rightarrow \tau^{ \pm} \nu_{\tau}$ branching ratio 18. At the Tevatron, CDF and D $\varnothing$ performed searches for $H^{ \pm}$produced in $p \bar{p} \rightarrow t \bar{t}$ where a top quark decays via $t \rightarrow H^{ \pm} b$ and the charged Higgs boson decays to $\tau \nu_{\tau}$. These searches have excluded the low and high $\tan \beta$ regions for charged Higgs masses up to $\sim 160 \mathrm{GeV}[19]$.

At the Large Hadron Collider (LHC) the search for the charged Higgs boson can also be performed at masses below the top quark mass. The main production mechanism is like at the Tevatron the top quark decay $t \rightarrow b H^{ \pm}$.

At LHC the search can also be extended to masses above the top quark mass. The main production mechanisms are the so-called $2 \rightarrow 3$ process $g g \rightarrow t b H^{ \pm}$and the $2 \rightarrow 2$ process $g b \rightarrow t H^{ \pm}$shown in Figure [1.1. Other production mechanisms have been considered, like pair production $g g, q \bar{q} \rightarrow H^{+} H^{-}$20] or associated production $q \bar{q} \rightarrow H^{ \pm} W^{\mp}$ [21], but their production cross sections are much lower. In addition, associated production suffers from large irreducible backgrounds 22 . Only the $2 \rightarrow 3$ and $2 \rightarrow 2$ processes have therefore been considered in this thesis. They partially overlap, so the inclusive cross section is obtained after a proper subtraction of the common terms 23 . The $H^{ \pm} t b$ vertex involved in both processes generates a coupling with a dependence on $\tan \beta$ which makes high and low values of $\tan \beta$ more accessible (for $\tan \beta \simeq 6-7$ the production cross section is minimal).

The couplings of the Higgs particles to fermions are proportional to the fermion mass [8], so they will decay preferentially into the heaviest 
particles allowed. Assuming that the typical mass scale of supersymmetric particles is much higher than the mass $m_{H^{ \pm}}$of the charged Higgs (i.e. $m_{\text {SUSY }} \geq 1 \mathrm{TeV}$ ), thus preventing the decay of charged Higgs bosons to supersymmetric particles, the $H^{ \pm}$has four dominating decay channels, as shown in Figure 1.2 $H^{ \pm} \rightarrow t b, H^{ \pm} \rightarrow \tau \nu_{\tau}, H^{ \pm} \rightarrow c s$ and $H^{ \pm} \rightarrow W^{ \pm} h$. The $H^{ \pm} \rightarrow c b$ channel, although it contains a $b$ quark much heavier than an $s$ quark, is suppressed through the CKM matrix coefficient involved in the intergenerational coupling $b \rightarrow c$.

The $H^{ \pm} \rightarrow t b, H^{ \pm} \rightarrow c s$ and $H^{ \pm} \rightarrow W^{ \pm} h$ decay channels have been previously studied for the ATLAS collaboration [24]. The $H^{ \pm} \rightarrow \tau \nu_{\tau}$ decay has also been studied for charged Higgs masses below the top quark mass [25].

This thesis focused on the study of the $H^{ \pm} \rightarrow \tau \nu_{\tau}$ decay channel for a charged Higgs heavier than the top quark in the context of the MSSM (see Section 1.2.2). It is shown that this channel has a significant discovery potential at LHC for large values of $\tan \beta$. Furthermore it has good sensitivity to the charged Higgs mass, allowing for its measurement. It also gives a handle on the determination of the value of $\tan \beta$. Results are reported in Papers III, IV and V.

\subsubsection{Minimal Supersymmetric Standard Model}

As discussed previously, the Standard Model has several problems. Supersymmetry (SUSY) is thought to have the potential to solve many of these issues.

The basic idea of Supersymmetry is to introduce a new symmetry between fermions and bosons. Each particle is assumed to have a supersymmetric partner, a sparticle, with the same quantum numbers except for the spin, so that each fermion has a bosonic spartner and each boson a fermionic one. Its simplest implementation (i.e. the most economical in new parameters, though it involves as much as at least 105 extra parameters [14) that encompasses the SM is called the Minimal Supersymmetric extension of the Standard Model (MSSM). In the Higgs sector (which is a 2HDM of type II), at tree level, two parameters suffice to describe the theory. They are usually chosen to be $\tan \beta$ and one of the Higgs masses, conventionally the mass $m_{A}$ of the CP-odd Higgs boson $A$.

If Supersymmetry were an exact symmetry, then particles would have the same mass as their superpartners. Since no supersymmetric particle has been observed so far, SUSY must be broken and sparticles are assumed to be so heavy that it has so far not been possible to produce them at currently available collider energies. How SUSY is broken is not known but many models exist where SUSY is broken in a "hidden" sector, and communicated to the model via a messenger, as with the Higgs field. Gauge mediated (GMSB), gravity mediated (SUGRA) or anomaly mediated (AMSB) SUSY breaking are some of the alternatives. In the MSSM, SUSY is broken "by 


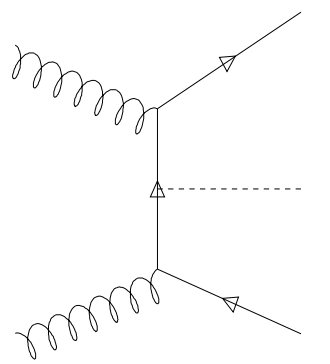

$\mathrm{gg} \rightarrow \mathrm{tH}^{+} \mathrm{b}$

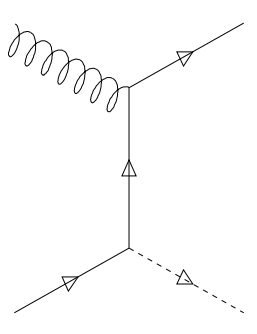

$\mathrm{gb} \rightarrow \mathrm{tH}^{+}$

Figure 1.1: The charged Higgs boson production at the LHC through the $2 \rightarrow 3$ process $g g \rightarrow t b H^{ \pm}$and the $2 \rightarrow 2$ process $g b \rightarrow t H^{ \pm}$.

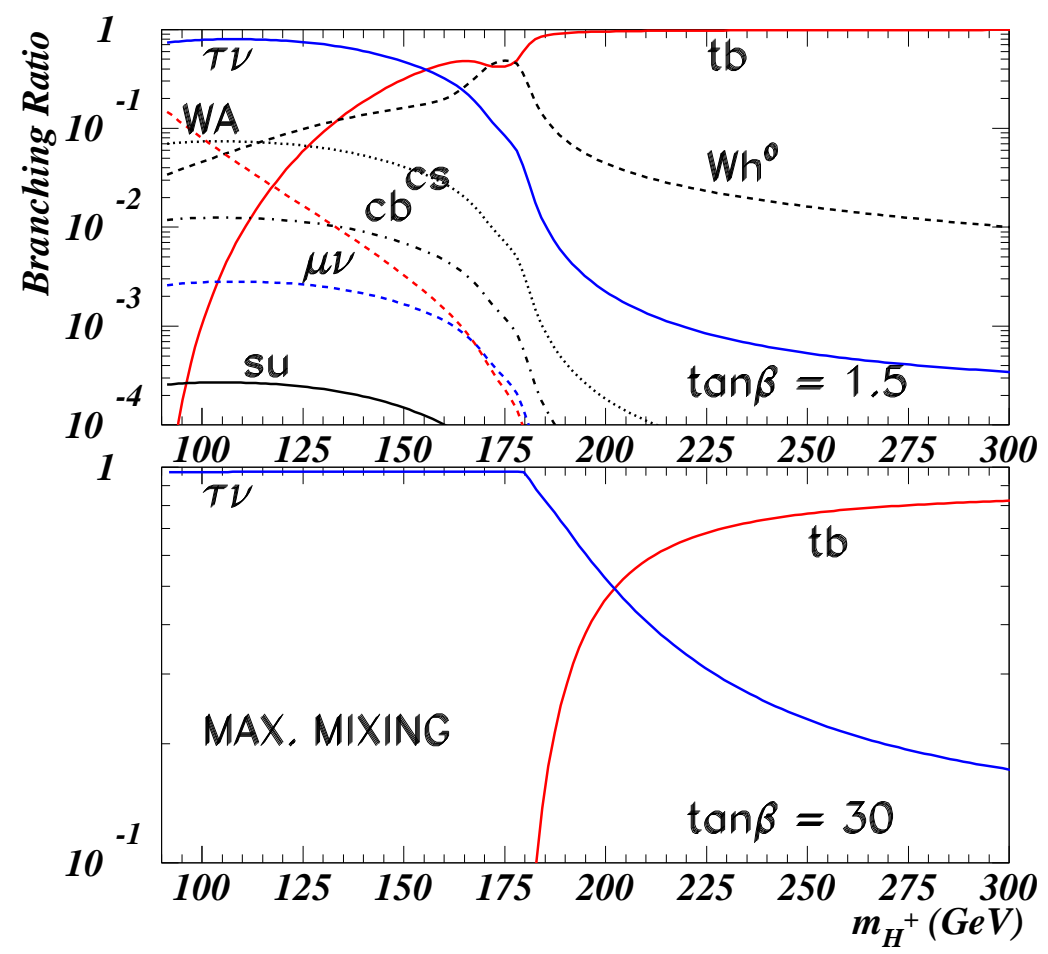

Figure 1.2: The branching ratios of the charged Higgs decays in SM particles as a function of $m_{H^{ \pm}}$for $\tan \beta=1.5$ (top plot) and $\tan \beta=30$ (bottom plot). 
hand" by introducing in the Lagrangian so-called soft SUSY breaking terms that are not invariant under SUSY.

One of the strong arguments for Supersymmetry is that it addresses the hierarchy problem, or rather the fine-tuning associated with the mass hierarchy. When trying to propagate SM results up to the Planck scale, radiative corrections to the mass of the Higgs boson are many orders of magnitude larger than the physical values of those masses [16. Although it would be mathematically possible to correct for this problem by imposing a tree-level value of the Higgs mass that is nearly equal and opposite to the correction, this seems very "unnatural". In Supersymmetry, equal numbers of fermions and bosons with identical couplings and loops of opposite signs automatically produce small corrections if their masses are comparable.

Moreover, when formulating a Grand Unified Theory (GUT) that unifies the three forces in a common description at some high energy scale Supersymmetry is one of the necessary ingredients: when evolving the three electroweak and strong coupling "constants" to higher energies within the framework of SUSY, they converge at an energy of around $10^{16} \mathrm{GeV}$ to a common value, while this is not the case in the SM. Finally, Supersymmetry is required when attempting to construct a common theory that includes gravity, a Theory Of Everything, that would describe all phenomena. 



\section{Chapter 2}

\section{ATLAS and the Large Hadron Collider}

The Large Hadron Collider (LHC) is the next particle accelerator to be built at CERN, the European Laboratory for Particle Physics near Geneva, Switzerland. It will bring protons into head-on collision at higher energies than ever achieved before to allow scientists to penetrate still further into the structure of matter and recreate the conditions prevailing in the Universe just $10^{-12}$ seconds after the "Big Bang". With such a high energy, physicists hope to find evidences of new physics, enabling them to answer some of the most profound questions about the nature of our Universe. The accelerator is described in Section 2.1 .

Four experiments, ATLAS, CMS (high luminosity proton-proton detectors), $\mathrm{LHCb}$ (optimised for the study of $\mathrm{CP}$ violation in $B$-meson decays) and ALICE (dedicated heavy-ion detector) will be run at the LHC. Only the ATLAS detector is within the scope of this thesis and presented in Section 2.2. Some of the physics results expected from the detector are discussed in Section 2.3 ,

\subsection{The next accelerator}

The Large Hadron Collider [26], scheduled to be completed and ready for the first physics run by 2007 , will deliver proton-proton collisions at an unprecedented centre-of-mass energy of $14 \mathrm{TeV}$.

The LHC will use the $27 \mathrm{~km}$ tunnel (100 $\mathrm{m}$ under ground) of the LEP collider which was decommissioned in 2001. There will be enough space left in the tunnel to also install in the future a lepton ring reusing LEP components which, operated jointly with the LHC, would provide electronproton collisions. The LHC will also run part of the time in heavy ion mode, colliding lead ions. All of this gives the LHC a very significant dis- 
covery potential, all the more so as its experiments are not only designed to look for theoretically predicted new physics, but also to reveal unforeseen phenomena.

It is a technological challenge to build such a machine: $7 \mathrm{TeV}$ of beam energy, an $8.3 \mathrm{~T}$ magnetic field in the dipoles and an instantaneous luminosity of $10^{34} \mathrm{~cm}^{-2} \mathrm{~s}^{-1}$ to cite only a few of the machine parameters. Two of the most difficult technological challenges are the superconducting magnets and the cryogenic systems.

Engineers had to design a special magnet following the two-in-one concept, where both magnetic channels are incorporated into a single iron yoke and cryostat, allowing the two proton beams to counter-rotate inside the same structure. The magnet coils are made of copper-clad niobium-titanium (NbTi) cables. NbTi is a classical superconductor, but to reach the expected magnetic field it is not sufficient to cool it with liquid helium at $4.2 \mathrm{~K}$ : LHC magnets will be operated at $1.9 \mathrm{~K}$, with superfluid helium.

Before reaching their colliding energy in the LHC ring, protons are accelerated by different machines. They are produced in a Duoplasmatron source and injected in a linear accelerator (Linac2) to reach $50 \mathrm{MeV}$. This beam is injected in the PS Booster synchrotron for further acceleration to $1.4 \mathrm{GeV}$, then in the PS (Proton Synchrotron) to reach $26 \mathrm{GeV}$. The final stage is the SPS (Super Proton Synchrotron) acceleration to $450 \mathrm{GeV}$ before insertion into the LHC machine which will bring protons to $7 \mathrm{TeV}$.

The energy available in the collisions at LHC is about 10 times higher than what has been achieved at LEP or the Tevatron (see Section 3.1). The luminosity will also be orders of magnitude higher, and will be achieved by filling each magnetic channel with 2,808 bunches of $10^{11}$ particles, separated from each other by only $25 \mathrm{~ns}$. Detectors installed at the LHC will therefore have to cope with this very high interaction rate in a very high radiation dose environment. The LHC will be run in two different luminosity modes: at first at 'low' luminosity $\left(10^{33} \mathrm{~cm}^{-2} \mathrm{~s}^{-1}, 10 \mathrm{fb}^{-1}\right.$ per year) among other things for $B$ physics, and then at 'high' luminosity $\left(10^{34} \mathrm{~cm}^{-2} \mathrm{~s}^{-1}, 100 \mathrm{fb}^{-1}\right.$ per year) to collect high statistics and study rare decays.

\subsection{The ATLAS detector}

The ATLAS experiment ( $A$ Toroidal $L H C A$ pparatu $S$ ) is currently being constructed by 1700 collaborators in more than 150 institutes from 35 countries around the world. It is a general-purpose detector which is designed to exploit the full potential of the LHC.

One of the major points of interest of the ATLAS physics program - and of many particle physicists - is the origin of particle mass, as mentioned in Chapter 1. This leads to a design optimised for the search of the Higgs boson. Other important goals are detailed studies of the top quark, the search for supersymmetric particles and heavy $W$ - and $Z$-like objects, for 
compositeness of 'fundamental' particles and investigation of CP violation in $B$ decays. Some of those subjects are addressed in Section 2.3.

Whereas particles that are to be observed in the experiment are infinitely tiny, the required detector is an enormous device: ATLAS has a radius of about $11 \mathrm{~m}$, a length of $42 \mathrm{~m}$ and an overall weight of 7000 tons!

The coordinate system of ATLAS is defined as follows: the beam direction defines the $z$-axis, the positive $x$-axis points from the interaction point to the centre of the LHC ring and the azimuthal angle $\phi$ is defined with respect to this axis, around the beam direction. The positive $y$-axis is pointing upward. The pseudorapidity is defined as $\eta=-\ln \left(\tan \frac{\theta}{2}\right)$ where $\theta$ is the polar angle with respect to the beam line. The pseudorapidity approximates the rapidity $y=\frac{1}{2} \ln \frac{E+p_{z}}{E-p_{z}}$ in the limit $m / E \rightarrow 0$ where $m$ is the particle mass.

The basic design considerations of the ATLAS detector can be summarised as follows [27: a powerful tracking system at high luminosity with the ability to measure particles with very low $p_{T}$, a very good electromagnetic calorimetry and missing transverse energy measurement capability, precise muon momentum measurements and a large acceptance in pseudorapidity.

In order to reach these requirements the ATLAS detector consists of four major components: the Inner Detector for tracking, the calorimeters (both electromagnetic and hadronic), the muon spectrometer and the magnet system. The latter consists of a very thin $2 \mathrm{~T}$ superconducting solenoid around the inner detector cavity, and large superconducting air-core toroids made of independent coils arranged with an eight-fold symmetry outside the calorimetry.

The Inner Detector's task is to reconstruct the tracks and vertices of each event, contributing to particle identification and supplying information about short-lived particles by detecting their decay vertices 28 . It is contained in a $7 \mathrm{~m}$ long cylinder of radius $1.15 \mathrm{~m}$, giving an acceptance of \pm 2.5 in pseudorapidity. Pattern recognition, momentum and vertex measurements and electron identification are achieved by combining a highresolution pixel detector (with 140 million detector elements) for the innermost layer followed by microstrip detectors, the SemiConductor Tracker, SCT (with 6.2 million readout channels) at inner radii with continuous tracking elements, the Transition Radiation Tracker (TRT), at outer radii. The TRT, made of straws, provides less spatial precision but more space points for tracking, while at the same time allowing for electron identification through the detection of transition radiation photons.

The electromagnetic (EM) calorimeter 29 should allow for electron reconstruction from $1 \mathrm{GeV}$ up to $3 \mathrm{TeV}$. An excellent energy resolution is required over a 10-300 $\mathrm{GeV}$ range to achieve a good mass resolution for Higgs boson decays to two photons or four electrons. It is a liquid argon detector with accordion geometry and lead absorber plates, preceded in the central region by a presampler detector to correct for energy loss before the 
calorimeter.

The hadronic calorimeter [30] was designed to identify jets and measure their energy and direction in order to allow a reliable jet-jet mass reconstruction, provide a good forward jet tagging and reconstruct the total missing transverse energy $\mathbb{E}_{T}$. The tile calorimeter consists of one barrel and two extended barrels made of iron absorbers and plastic scintillating plates as active material, read out with wavelength shifting fibres. Liquid argon is used to detect the ionisation in the end-cap hadronic calorimeter and in the forward calorimeter. Both of them are housed in the same cryostat as the EM end-caps. End-caps and the first layer of the forward calorimeter contain copper plates while the other two are made of tungsten.

\subsection{ATLAS physics potential}

The design of the ATLAS detector was motivated by physics, though moderated by financial and technical considerations. At all stages, from the early design to the ongoing construction and commissioning, and after 2007 during data taking and analysis, physicists rely on different techniques to simulate the expected behaviour of the detector. Some of the techniques that are of relevance for the work presented in this thesis are reviewed in Section 2.3.1 and physics results expected from the detector are briefly described in Section 2.3.2.

\subsubsection{Detector simulation}

In order to have an idea of what the detector output would look like for a certain physics process, several stages are involved. It starts with the use of a Monte Carlo program to generate proton-proton collision events, i.e. sets of outgoing particles produced in the interaction between the two incoming protons. The program used for the ATLAS physics studies presented in this thesis is Pythia 5.7 [31.

The produced final state particles are then passed through a simulation program that will generate the same kind of data as the real detector would produce in response to the particles traversing its different components. The next step is data reconstruction: from the output data of the different subdetectors are generated so-called physics objects like electrons, muons, taus, jets, missing energy, etc. It remains to analyse this data and devise a strategy to recognise the originally generated physics process and discriminate it from the background.

Two different ways of producing physics objects from Monte Carlo events are presented in the following sections. 


\section{Full simulation}

Most studies presented in Papers I and II were performed using GEANT3 32 to simulate the detector. This software takes as an input the particles produced by a Monte Carlo generator and describes their passage through matter. It has to contain the geometry of the detector, its different subdetectors and modules, their physical properties like chemical composition and density. It then simulates the transport of the input particles through the various regions of the detector, taking into account the geometrical volume boundaries and all physical effects due to the nature of the particles themselves, to their interactions with the different materials and to the magnetic field.

Finally it records elements of the particle trajectories and responses from the sensitive detectors in the form of hits or energies. Those responses are digitised to produce an output similar to the readout system, adding electronic noise. The information available is now of the same form as the raw data that would come out of the detector during data taking.

To make sense of these data a reconstruction program is run. It forms jets out of calorimeter energy clusters, reconstructs muon tracks in the muon system and electrons in the EM calorimeter, etc. It also has pattern recognition capabilities to find tracks in the Inner Detector. For the studies presented in this thesis the XKALMAN pattern recognition and track fitting package was used [33]. It looks for possible track candidate trajectories in the TRT and attempts to prolong them layer by layer towards the innermost part of the tracker, taking into account multiple scattering and bremsstrahlung losses along the way. A global fit is finally performed to provide track parameters.

All steps described in this section are performed when a study is made in the 'full simulation' environment. It aims at giving as close a picture of reality as possible, mimicking details of the geometry and physical properties of the detector and looking blindly at its output. When reconstructing the information, no prior knowledge of the originally generated particle is assumed (the information is kept, though), similarly to the situation when real data is taken.

\section{Fast simulation}

Although full simulation is the most realistic approach to detector simulation, it is not without drawbacks. The generated data outputs are huge, especially in the intermediate steps of simulation and digitisation, and the whole process is time consuming even on today's fast computers - the CPU time necessary to process one full event does not seem to decrease over the years while computers get more powerful, since apparently the simulation refinement is increasing at the same pace.

Generating millions of such events for analysis is therefore often impractical. This is the reason why a 'fast simulation' was introduced. Instead 
of simulating the interactions of each and every particle with matter in the detector, each kind of physics object was studied using full simulation. The detector response to the different objects was then parameterised as a function of their momentum or pseudorapidity for instance, and all those parameterisations were put into a common software package called ATLFAST 34. An extension of the package simulates efficiencies and rejections for tagging of $b$-jets, $c$-jets and $\tau$-jets. The Monte Carlo type of each particle is used to apply the corresponding parameterisation, unlike in the full simulation case. Hence it is possible to get a reasonable idea of what the event would really look like, without spending too much computational resources on producing fully simulated results.

Fast simulation was used in Papers III, IV and V, and partially in Paper II (to simulate the background). The relevance of fast simulation is described and discussed in Paper II and in Reference 34. A study to parameterise an effect for fast simulation using full simulation results was performed in Paper I.

\subsubsection{Physics results}

Using techniques described in Section 2.3.1 several physics processes were generated for this thesis, showing the large range of processes that can be studied with the existing tools. Paper I focuses on $\tau$ lepton detection and $\tau-\tau$ invariant mass calculation, of relevance especially to test the existence of Supersymmetry. Paper II investigates the ATLAS capabilities in $B$ physics and $\mathrm{CP}$ violation measurement, providing an important test of the Standard Model. Papers III, IV and V explore the ATLAS discovery potential for a charged Higgs boson and the precision of measurements it can reach on parameters crucial to the understanding of physics beyond the Standard Model (if it exists). The papers are summarised in Chapter 4 and constitute an integral part of this thesis. 


\section{Chapter 3}

\section{Triggering with the $D \varnothing$ detector}

The $\mathrm{D} \varnothing$ experiment is, together with CDF, one of the two experiments studying proton-antiproton collisions at the Tevatron collider which is located at Fermilab (the Fermi National Accelerator Laboratory, FNAL) near Chicago, Illinois, USA. The experiment took data from 1992 through the beginning of 1996 at a centre-of-mass energy of $1.8 \mathrm{TeV}$, with an integrated luminosity of approximately $125 \mathrm{pb}^{-1}$ in the so-called Run 1 . Among other interesting results, the data analysis in D $\varnothing$, together with that in CDF, led to the discovery of the top quark in 1995 335. Since then, the accelerator and the experiments have undergone major upgrades, and data taking started again in March 2001. This second period of activity is called Run 2.

The Tevatron accelerator complex is described in the first section of this chapter. Then the $\mathrm{D} \varnothing$ detector is presented with all its subdetectors. Finally the trigger system is described along with the author's contribution to the development of triggers for $Z \rightarrow \tau \tau$ and $W^{ \pm} \rightarrow \tau \nu_{\tau}$ events.

\subsection{The Tevatron accelerator}

The Tevatron is, until the foreseen LHC startup in 2007 (see Section 2.1), the accelerator that delivers the highest collision energy in the world. It collides protons and antiprotons at a centre-of-mass energy of $1.96 \mathrm{TeV}$. When it was built in 1983 it was the first superconducting synchrotron. A magnetic field of $4.2 \mathrm{~T}$ bends the particles around the $1 \mathrm{~km}$ radius tunnel.

In Run 2 the instantaneous luminosity should reach $2 \cdot 10^{32} \mathrm{~cm}^{-2} \mathrm{~s}^{-1}$, although it is at present (August 2003) only achieving up to $4.5 \cdot 10^{31} \mathrm{~cm}^{-2} \mathrm{~s}^{-1}$. To generate this high luminosity, 36 bunches of protons and antiprotons circulate in the machine, grouped in three superbunches, and separated by 396 ns. During the second phase of Run 2 (Run 2b), which will correspond 
to data taken after the ongoing Run 2a (expected to accumulate approximately $2 \mathrm{fb}^{-1}$ ), the accelerator and the detectors will undergo another upgrade to reach the ultimate luminosity.

The Tevatron is the last stage of a long acceleration chain. First, negative hydrogen ions $\left(\mathrm{H}^{-}\right)$are accelerated by a $750 \mathrm{kV}$ Cockcroft-Walton accelerator, bunched and fed into a linear accelerator (LINAC) that brings the ions to an energy of $400 \mathrm{MeV}$. Ions meet a carbon foil that strips both electrons and leaves only protons that are injected in the Booster, a 475 meter long synchrotron that brings protons from $400 \mathrm{MeV}$ to $8 \mathrm{GeV}$ and delivers them to the Main Injector. This accelerator will bring protons to $150 \mathrm{GeV}$ for injection into the Tevatron, and to $120 \mathrm{GeV}$ for antiproton production. This beam is run on to a nickel target, producing many secondary particles, including antiprotons. The produced antiprotons are stored in the Accumulator before being inserted in the Main Injector which accelerates them to $150 \mathrm{GeV}$.

Now both $150 \mathrm{GeV}$ proton and antiproton beams are injected in the Tevatron where they are accelerated to $980 \mathrm{GeV}$. The beams will collide at two interaction points surrounded by the $\mathrm{CDF}$ and $\mathrm{D} \emptyset$ particle detectors. The $\mathrm{D} \emptyset$ detector is described in the next section.

\subsection{The $\mathrm{D} \emptyset$ detector}

Like most particle physics detectors, $\mathrm{D} \emptyset$ is made up of several subdetectors which are described briefly in the following sections, from the innermost silicon detector to the forward proton detectors. Emphasis is put on the parts that are crucial to the trigger studies presented later, namely the calorimeter and the fibre tracker.

The $\mathrm{D} \emptyset$ coordinate system is defined as follows: the direction of the proton beam defines the positive $z$-axis, the positive $x$-axis points horizontally outward from the centre of the Tevatron and the $y$-axis is pointing up.

\subsubsection{The tracking system}

One of the major upgrades of the $\mathrm{D} \emptyset$ detector for Run 2 is the introduction of a $2 \mathrm{~T}$ magnetic field provided by a $2.8 \mathrm{~m}$ long, $60 \mathrm{~cm}$ radius superconducting solenoid magnet. This allows the measurement of charged particle momenta from the curvature of their trajectory in the magnetic field. The trajectories are recorded in two tracking devices located inside the solenoid: the Silicon Microstrip Tracker (SMT) and the Central Fiber Tracker (CFT).

The silicon tracker [36 uses microstrip detectors providing a hit resolution of approximately $10 \mu \mathrm{m}$. The detectors are mounted on four layers in the central barrel with a mixture of single-sided axial detectors and double-sided $90^{\circ}$ and $2^{\circ}$ stereo detectors. Forward disks with double-sided stereo detectors complement the barrel. All detectors are radiation hard and mounted on beryllium bulkheads which serve both as a mechanical support 
and as a coolant provider. The SMT allows tracking out to $|\eta|<3$ and has nearly 800,000 readout channels. It has the highest resolution within the tracking system and allows good momentum measurement and vertex reconstruction (both primary and secondary).

The scintillating fibre tracker [37] surrounds the silicon detector and covers the central pseudorapidity region within the range $\eta= \pm 1$. It consists of 8 layers of two fibre doublets. Each fibre doublet is made of two layers of $835 \mu \mathrm{m}$ diameter scintillating fibres separated by $870 \mu \mathrm{m}$. One layer is offset by half the fibre spacing with respect to its partner, removing all gaps. On each of the eight layers there is an axial doublet and a stereo doublet at $\pm 2^{\circ}$. The eight axial layers are also used for triggering, as described in Section 3.3

The scintillating fibres are up to $2.5 \mathrm{~m}$ long, and mounted on carbon fibre support cylinders. The light from the fibres is piped out by clear fibres and converted into electrical pulses by visible light photon counters, VLPCs, in a cryostat outside the tracking volume. VLPCs are small silicon devices operated at $9 \mathrm{~K}$ with an excellent quantum efficiency and high gain. In total the CFT has approximately 77,000 readout channels. The hit resolution is about $100 \mu \mathrm{m}$.

\subsubsection{The preshower detectors}

The tracking system is complemented by central and forward preshower detectors (CPS and FPS). The CPS [38] is mounted on the solenoid $(|\eta|<1.2)$ and the FPS [39] sits on the end calorimeter cryostats (see below) and covers $1.4<|\eta|<2.5$. Both detectors consist of a lead absorber followed by several layers of axial and stereo triangular scintillator strips with wavelengthshifting fibres read out by VLPCs. The detectors function as a tracker by providing precise position measurements, but also as a calorimeter by early energy sampling. They improve electron identification and triggering and correct for energy loss in the solenoid.

\subsubsection{The calorimeter system}

The Run 2 calorimeter is the same as in Run 1 [40]. The readout electronics had to be upgraded to cope with the higher rates 41, but the detector itself is unchanged. It is a hermetic, stable, radiation-hard liquid argon sampling calorimeter. It uses depleted uranium as a primary absorber in the electromagnetic and the inner hadronic sections because of its high density (hence a compact design) and compensating $e / \pi$ response. Stainless steel and copper are used in the outer layers.

The calorimeter is made up of three units, each contained in its own cryostat (see Figure 3.1): the central calorimeter (CC) and the two end calorimeters (EC). The CC consists of three concentric cylindrical sections of modules, covering up to $|\eta|=1.2$ : the four electromagnetic layers (EM) first, followed by three fine hadronic $(\mathrm{FH})$ and one coarse hadronic $(\mathrm{CH})$ 
layers. The EC is made of four EM layers, covering $1.4<|\eta|<4$, and three concentric cylinders for hadronic showers: the inner, middle and outer modules ( $\mathrm{IH}, \mathrm{MH}$ and $\mathrm{OH})$.

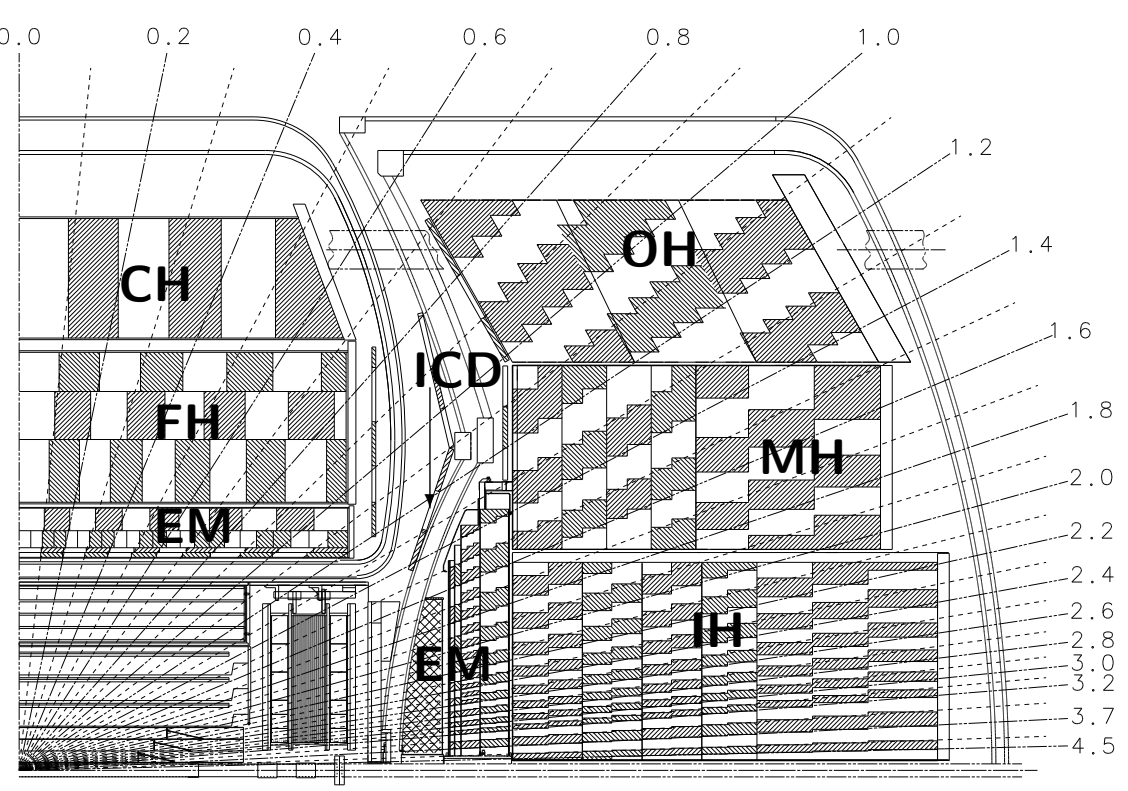

Figure 3.1: Side view of a quarter of the DØ calorimeter.

All layers are segmented into cells of size $0.1 \times 0.1$ in $\eta \times \phi$ space except for large pseudorapidity $(|\eta|>3.2)$ where cells would be too small (and are therefore $0.2 \times 0.2$ in size), and in the third EM layer. In this section of the calorimeter electrons are expected to reach the shower maximum so the granularity is increased to $0.05 \times 0.05$ to allow a better measurement. All modules are arranged into semi projective towers and their boundaries are not aligned to avoid continuous inter-module cracks. For triggering purposes, cells are summed in $0.2 \times 0.2$ towers and the information sent through a separate readout (see Section 3.3 for details).

At the overlap between the CC and EC $(1.1<|\eta|<1.4)$ the Inter Cryostat Detector (ICD 42) is installed to compensate for the dead region between the cryostats and maintain performance in presence of the magnetic field and additional material from the solenoid. It provides a single energy sample in this region that improves the jet energy and $\mathbb{E}_{T}$ measurements. ICD cells are aligned with the pseudo projective towers in the EC, with the same segmentation. They consist of plastic scintillators read out by phototubes.

The signal from each calorimeter cell, proportional to the energy deposited by particles in the active media, is carried outside the cryostat via coaxial cables to preamplifiers that integrate the charge from the calorim- 
eter cells to produce voltages. These pulses are transmitted to a baseline subtractor (BLS). The BLS treatment removes slowly varying offsets in the input voltage by sampling the signal before and after the bunch crossing, the difference providing only the signal amplitude. The signal is finally read out and digitised by analog to digital converters.

The upgraded electronics allows the calorimeter to cope with the increased luminosity and reduced bunch spacing by reducing the shaping and readout time. Analog buffering was implemented to store data while waiting for a trigger decision. The new electronics has the drawback of introducing more electronic noise than in Run 1 . Nevertheless the calorimeter high linearity and energy resolution are preserved. Calibration, both online with calibration pulses and offline with known physics processes, is crucial.

Understanding the details of the electronic response is a still ongoing process. One aspect of it is the choice of the zero-suppression threshold, i.e. the cell energy below which it is not read out. The impact of zero suppression on the trigger was studied in Paper VI. A dedicated task force has been working on understanding all the effects adversely affecting calorimeter data and reconstructed objects, but a number of major issues remain unresolved.

\subsubsection{The muon spectrometer}

The muon system is divided into the wide angle muon spectrometer (WAMUS) which covers the central detector $(|\eta|<1)$ and the forward angle muon spectrometer (FAMUS) in the range $1<|\eta|<2$. Both subsystems are located inside a $1.8 \mathrm{~T}$ toroid field created by a solid-iron magnet.

The WAMUS 43 consists of three subdetectors. Three layers (A, B and $\mathrm{C}$ ) of proportional drift tubes (PDTs) provide muon identification and a momentum measurement independent of the central tracker. The A-layer is between the calorimeter cryostat and the muon toroid magnet, while the $\mathrm{B}$ and $\mathrm{C}$ layers sit outside the magnet, $1 \mathrm{~m}$ apart from each other. The second subdetector is the A- $\phi$ counters, a layer of scintillators between the calorimeter and the A-layer for triggering and rejection of out-of-time cosmic rays and scattered particles. Finally the cosmic caps cover the top and sides (and part of the bottom) of the muon detector to reject cosmic rays.

The FAMUS [44] also has three subsystems: three layers of mini-drift tubes (MDTs) for momentum measurement, three layers of scintillating material mounted on each layer of MDTs for timing and cosmic rejection, and shielding around the beam pipe to reduce trigger rates, fake track reconstruction and ageing of the detectors.

\subsubsection{The forward proton detector}

The forward proton detector (FPD) is designed to study diffractive processes by measuring protons and antiprotons that have scattered at small angles [45]. It consists of spectrometers formed by Roman pots and the 
Tevatron magnets, situated approximately $30 \mathrm{~m}$ away from the interaction point. The Roman pots are stainless steel containers used to insert scintillating fibre detectors close to the beam to measure the coordinates of proton and antiproton tracks, thus allowing the reconstruction of the track momentum and scattering angle.

\subsubsection{The luminosity monitor}

In order to normalise physics analyses and measure cross sections, an accurate knowledge of the delivered luminosity is required. This is done using a specialised detector, the luminosity monitor (LM) [46. Two hodoscopes, made of plastic scintillators with photomultiplier tubes are placed on the inner face of the end calorimeter cryostats. They cover the pseudorapidity region $2.7<|\eta|<4.4$.

The luminosity is measured by identifying beam crossings containing non-diffractive inelastic interactions, looking for coincidences between the proton and antiproton bunches. The time difference between signals produced by the north and south detectors allows the differentiation between collisions (luminosity) and beam losses (halo): a proton from the beam will go through the north detector first, and 9 ns later through the south LM, while antiprotons do the opposite. But particles produced at the D $\varnothing$ interaction point will reach both LM at approximately the same time, the time difference allowing a fast measurement of the vertex position of the interaction. All the information is sent to the trigger framework and used to take the trigger decision. The full trigger scheme is presented in the next section.

\subsection{The D $\varnothing$ trigger system}

In order to get as many events as possible on tape for analysis in the $D \varnothing$ experiment the luminosity delivered by the Tevatron should be as high as possible. The design bunch spacing of $132 \mathrm{~ns}$ implies a $p \bar{p}$ collision rate of $7.6 \mathrm{MHz}$. With a mean $250 \mathrm{kB}$ of data per event on tape, that would represent of the order of $2 \mathrm{~TB}$ of data per second if every single bunch crossing were to be recorded, an amount clearly impossible to deal with, both technically and financially. The experiment must therefore focus on "interesting" events to reduce this data flow to a more manageable level (fortunately, most of the events have no real interest from a physics analysis point of view). It was decided that the maximum rate to tape should be of the order of $50 \mathrm{~Hz}$, or roughly $12 \mathrm{MB}$ of data per second.

In order to select 50 events out of 7.6 million crossings, a complex system is in place, the $D \varnothing$ trigger system, to filter out interesting events and suppress background. Given a ten-fold increase in luminosity, the trigger system had to be upgraded from the Run 1 trigger [47. It consists of three levels, described in the following sections. 
The trigger system is complex and the physics output of the experiment relies heavily on it. Therefore much effort over the duration of this thesis was spent by the author to better understand it and to make it more understandable, more reliable and more accessible to other physicists. This led to lots of fixes and patches at all three levels of the whole trigger system, in combination with physics oriented studies to design trigger strategies for specific physics channels involving $\tau$ leptons in their final state (namely $Z \rightarrow \tau \tau$ and $\left.W^{ \pm} \rightarrow \tau \nu_{\tau}\right)$. Most of this work is hard to document as it essentially results in corrections of previous errors and bugs in the trigger software leading to that the experiment behaves in the way it is intended to. On the other hand, such work is essential to the experiment, enabling the whole collaboration to get meaningful results.

Most of these studies require the use of a trigger simulator, D0TRIGSIM, which emulates the behaviour of Level 1 and Level 2 hardware and has an offline interface to the software used online for Level 2 and Level 3 [48]. It can be run on Monte Carlo samples to design a trigger, or on data to validate trigger performances.

\subsubsection{Level 1 trigger}

The Level 1 trigger (L1) is a hardware and firmware system constructed with the aim to reduce the input rate of $7.6 \mathrm{MHz}$ to around $10 \mathrm{kHz}$ for input into the Level 2 trigger (right now this rate is between 1.5 and $2.5 \mathrm{kHz}$ ). It consists of the L1 trigger framework (L1FW) and several trigger subsystems presented below. A schematic view of the trigger is shown in Figure 3.2 .

\section{Level 1 trigger framework}

Each L1 subsystem processes data from the detector and sends, for each beam crossing, a set of binary inputs, so-called And-Or terms, to the L1 framework [49]. A decision being taken in $4.2 \mu \mathrm{s}$ (and not between beam crossings) a pipeline able to retain 32 crossings will ensure deadtimeless operation. The And-Or terms can contain physics information from the subsystems, but also status reports from different sources like readiness of the data acquisition system (DAQ), beam conditions, cosmic background veto, or any other information needed to form a L1 decision.

There is a maximum of 256 And-Or terms, and the L1FW can make up to 128 combinations of those bits. If any one of these And-Or combinations is satisfied ("fired"), it means that this bunch crossing has all the characteristics that this particular L1 trigger is looking for. If the DAQ is ready for the next event (i.e. if no other condition forbids the exposure of this trigger), the Specific Trigger is fired, a Level 1 accept decision is issued and the event is sent to Level 2 for further processing.

It may happen that a specific trigger is satisfied too often (either by construction or because of a detector problem), leading to too high an accept rate for the available readout bandwidth. This is addressed by using a 


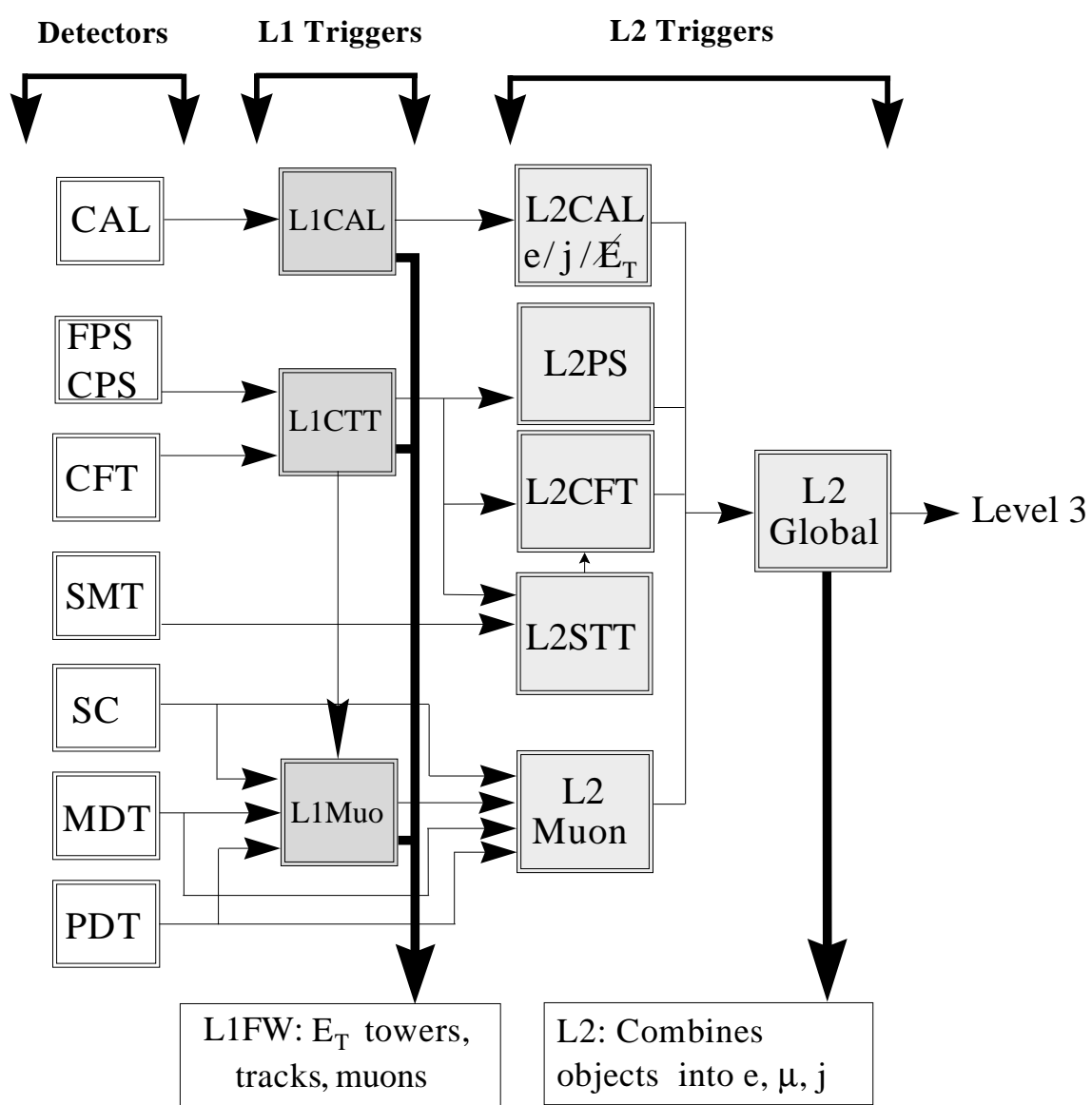

Figure 3.2: The DØ Level 1 and Level 2 trigger. 
prescaler, that will allow a fired And-Or combination to generate a L1 accept (firing the corresponding Specific Trigger) only a fraction of the time: even if the And-Or combination would allow the trigger to pass, it will be exposed only according to the prescaler rate.

All parameters necessary for the trigger system to perform its tasks are provided to the framework via a trigger list which describes the different And-Or terms, Specific Triggers and general parameters.

\section{Calorimeter trigger}

The Level 1 calorimeter trigger (L1CAL) uses information from the calorimeter (as mentioned in Section 3.2.3) in the form of $0.2 \times 0.2$ towers in $\eta \times \phi$, representing 32 divisions in $\phi$ and 40 in $\eta$ for a total of 1280 towers [50]. Only the electromagnetic and hadronic sections are used, the coarse hadronic layer being too noisy for meaningful trigger decisions. Currently only $\eta$ bins running from 4 to 35 are used (i.e. $|\eta|<3.2$ ) as the last towers are either not yet commissioned or debugged. The still ongoing commissioning of the trigger is a long process: in June 2002, after more than a year of data taking with a maximum pseudorapidity of 0.8 , the coverage went to 2.4 and in March 2003 up to 3.2. It is expected to reach $|\eta|<4$ in the future.

The information available for trigger decision is the transverse energy $E_{T}$ in each tower in the EM part and in total (EM plus hadronic). It is digitised in $0.25 \mathrm{GeV}$ bins. $E_{T}$ values can then be compared to reference sets (refsets), that is, specific $E_{T}$ thresholds provided to the trigger framework through the trigger list. It is then possible to count how many towers have EM or total energy above a certain threshold, and select events on this criterion. Such terms are called $\operatorname{CEM}(\mathrm{n}, \mathrm{X})$ and $\mathrm{CJT}(\mathrm{n}, \mathrm{X})$ for $\mathrm{EM} E_{T}$ and total $E_{T}$ respectively, where $\mathrm{n}$ is the minimum number of towers above $E_{T}=\mathrm{X} \mathrm{GeV}$. For instance a particular event will fire $\operatorname{CJT}(1,5)$ if there is at least one tower with $E_{T}>5 \mathrm{GeV}$. Framework limitations require a small number of refsets. For example the current (August 2003) trigger list global_CMT-12.20 has EM thresholds 3, 6, 9 and $11 \mathrm{GeV}$ and jet thresholds 3,5 and $7 \mathrm{GeV}$.

At some point, the system should also be able to use global variables to trigger on, such as the total energy and the missing transverse energy in an event. Those features are not yet fully commissioned, although highly desirable.

L1 trigger efficiency curves are determined by plotting the fraction of events passing the trigger condition as a function of the reconstructed jet/EM object $E_{T}$. Such curves can be seen in Figure 3.3 .

In order to determine a trigger strategy for $Z \rightarrow \tau \tau$ events, such studies were also performed. One of the $\tau$ 's decays leptonically and the other to hadrons. An extra difficulty in the context of $\tau$ physics at $\mathrm{D} \varnothing$ is that $\tau$ leptons have only very recently been observed, owing to those trigger studies [52. It was therefore impossible to produce turnon curves based on 

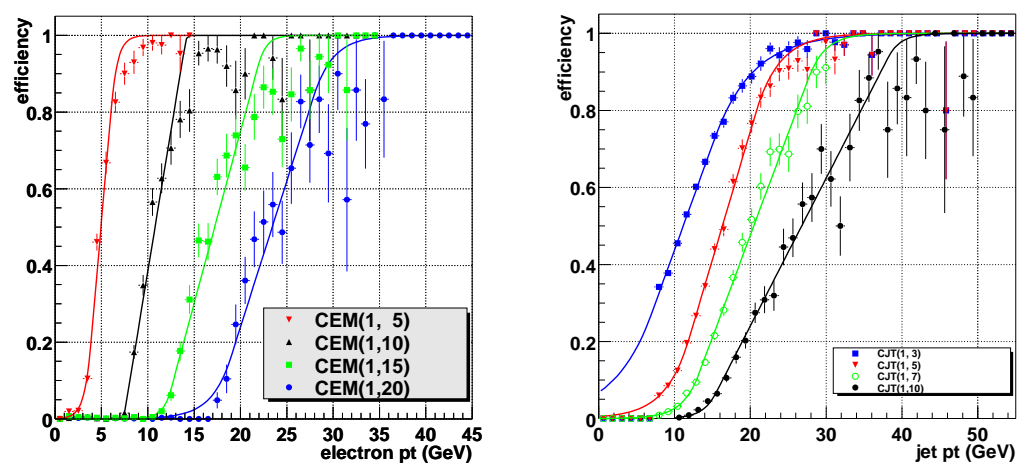

Figure 3.3: Level 1 calorimeter $\operatorname{CEM}(1, \mathrm{X})$ and $\mathrm{CJT}(1, \mathrm{X})$ turnon curves. An unbiased sample of events triggered by a muon trigger was used. The left-hand plot shows the turnon for CEM triggers at different $E_{T}$ thresholds with respect to an offline electron $p_{T}$. The right-hand plot represents CJT turnons with respect to the reconstructed jet $p_{T}$. In both cases the turn on is slower with higher $E_{T}$ thresholds (from [51]).

real reconstructed taus, as is the case for real electrons or jets in Figure 3.3. Neither could good criteria be derived for reliable $\tau$ identification until real taus are observed in sufficient numbers to study their properties at DØ.

Instead, the Monte Carlo visible transverse momentum of hadronic taus, i.e. the $p_{T}$ of all decay products except the neutrino, is used as a reference. Events are processed through the trigger simulator to see the expected behaviour of L1CAL when a $\tau$ lepton coming from a $Z$ boson interacts in the calorimeter. Results can be seen in Figure 3.4. It shows what the challenge is for triggering on $\tau$ objects: although the $\tau p_{T}$ distribution peaks around $40 \mathrm{GeV}$ (plot (a)), what can actually be seen in the calorimeter is only the visible $p_{T}$, shown in plot (b). When the trigger coverage was limited to $|\eta|<0.8$ this seriously limited the ability to trigger on $Z \rightarrow \tau \tau$ events, as only a $50 \%$ efficiency could be reached even for high energy taus (plot (c)). Increasing the acceptance to 2.4 it looks much better but the slow turn on, comparable to what is shown in Figure 3.3 has a high price for the overall efficiency on the signal.

Nevertheless it was decided to run a CJT $(1,5)$ trigger to select those events, as the rate of $\mathrm{CJT}(1,3)$ would have been too high (hence necessitating a L1 prescale). It was combined with a single muon trigger (resp. electron trigger $\operatorname{CEM}(1,10))$ to select $Z \rightarrow \tau(\mu) \tau($ had) $($ resp. $Z \rightarrow \tau(e) \tau($ had $)$ ) events. 

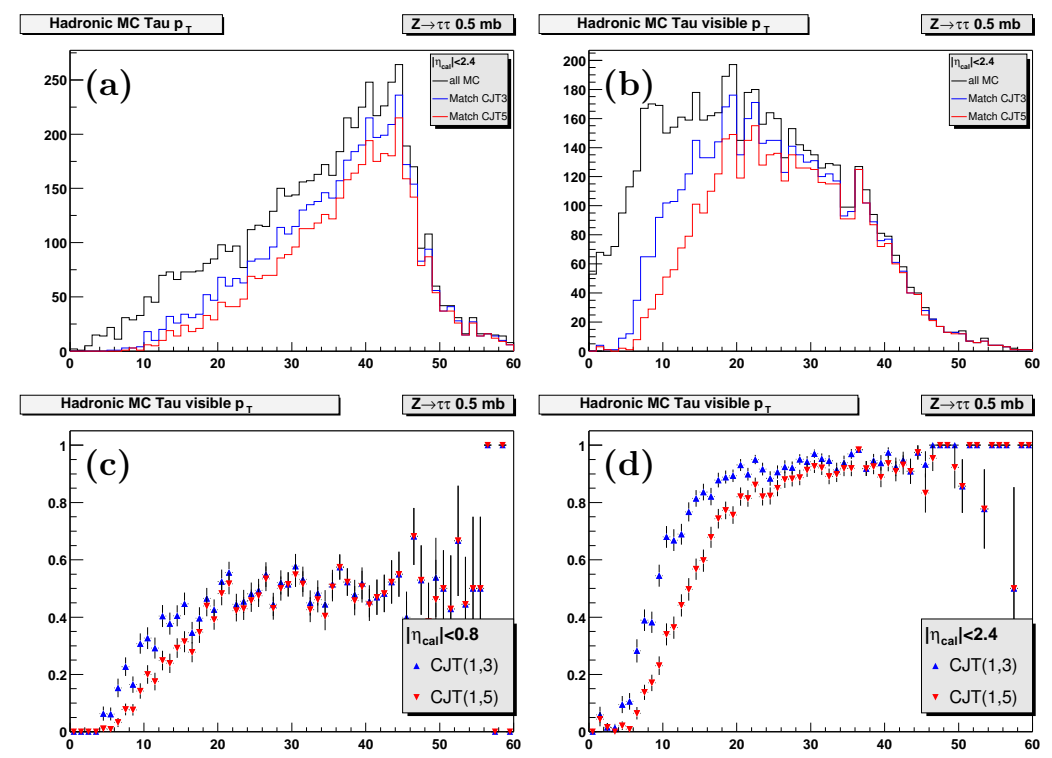

Figure 3.4: Level 1 calorimeter triggers $\mathrm{CJT}(1, \mathrm{X})$ on hadronic taus from $Z \rightarrow \tau \tau$ decays. The tau $p_{T}$ is shown on plot (a), and the visible part (removing neutrinos) of the tau $p_{T}$ on plot (b). The black curve is all hadronic taus, the blue (red) curve only those matched to a $3 \mathrm{GeV}(5 \mathrm{GeV})$ trigger tower. Plots (c) and (d) represent turnon curves for CJT(1,X) for a trigger coverage $|\eta|<0.8$ and $|\eta|<2.4$ respectively.

\section{Muon trigger}

The Level 1 muon trigger (L1Muo) is divided in three geographical regions: central, north and south [53. It takes inputs from the WAMUS and FAMUS hardware (both scintillation counter hits and wire chamber centroids). The algorithm uses PDT and MDT centroids verified by an accompanying scintillator hit. This confirmation is necessary because the drift time in the PDTs $(\sim 500 \mathrm{~ns})$ is greater than the bunch crossing time, so that centroids can originate from several crossings. This is not the case for MDTs, with a drift time of $\sim 80 \mathrm{~ns}$, but it helps rejecting background particles not produced at the interaction point.

L1 muons are reported according to their $p_{T}$ (above four different thresholds: 2, 4, 7 and $11 \mathrm{GeV}$ ), pseudorapidity region and object quality (loose, medium or tight). The quality reflects how many different muon detectors or layers are used in forming the candidate muons. A low $p_{T}$ trigger muon only has centroids found in the A-layer while high $p_{T}$ muons have correlations between the A-layer and the B- or C-layer. For high $p_{T}$ tracks penetrating the iron toroid, the cosmic cap veto scintillators are also used.

The L1Muo can also operate in collaboration with the L1 track trigger 
described in the next section. This is the only case where information from different subdetectors can be combined at Level 1. It matches CFT tracks with scintillator hits. This method gives a better $p_{T}$ measurement but has been delayed by the L1 tracking commissioning.

Level 1 muon trigger terms are referred to as $\operatorname{MUO}(\mathrm{n}$, pt, eta, scint, wire, option) where $\mathrm{n}$ is the number of required muons (up to three), $\mathrm{pt}$ is the $p_{T}$ threshold, eta is the pseudorapidity coverage, scint is the scintillator quality, wire the wire quality and option any additional parameter (like same-sign tag for dimuon triggers).

To trigger on the channel $Z \rightarrow \tau(\mu) \tau($ had) a single muon trigger was used in combination with the calorimeter trigger CJT(1,5). It requires at least one tight scintillator muon in the detector. The wires were not used as they were not yet commissioned and no $p_{T}$ requirement was imposed because its measure is not good enough when using scintillators only. The complete L1 trigger for this channel is therefore a calorimeter jet above $5 \mathrm{GeV}$ and a tight scintillator muon.

\section{Track trigger}

One of the major upgrades of the $\mathrm{D} \emptyset$ trigger system for Run 2 is the addition of the Level 1 central track trigger (L1CTT) to increase rejection [47. The L1CTT system [54] uses data from three subdetectors: the CFT (axial fibres only), the CPS (axial strips only) and the FPS (see Section 3.2 for detector details).

The L1CTT system is designed to trigger on charged particles with $p_{T}>1.5 \mathrm{GeV}$ and to find preshower clusters and match them to found tracks. It provides track information to the L1 muon system and to Level 2 preshower and tracking subsystems.

All fibres are arranged in eighty $4.5^{\circ}$ trigger sectors in the transverse plane and their output signal is discriminated. Each sector corresponds to a particular board that unpacks the CFT data and compares the fibre hits with approximately 20,000 predefined analytically generated track equations in order to form coincidences from hits in all eight layers. Such a track and its hit pattern are shown in Figure 3.5. Found tracks are organised into four transverse momentum levels, the $p_{T}$ bins, corresponding to tracks with $p_{T}$ in the ranges $1.5-3 \mathrm{GeV}, 3-5 \mathrm{GeV}, 5-10 \mathrm{GeV}$ and above $10 \mathrm{GeV}$.

Each sector can report up to six tracks in each $p_{T}$ bin. Additional information is also available like matching preshower clusters and the total number of doublet hits in the CFT (the sector occupancy). It also reports whether a track is isolated, meaning that it is the only track in a sector and that there is no track in the two adjacent sectors.

When the CTT is fully commissioned, track information will also be summed up by octants (eight groups of ten sectors) and by quadrants (four groups of two octants).

All L1CTT trigger terms planed to be implemented are defined in Reference [55] and reported in Table 3.1] Only the TTK and TIS terms (tracks 


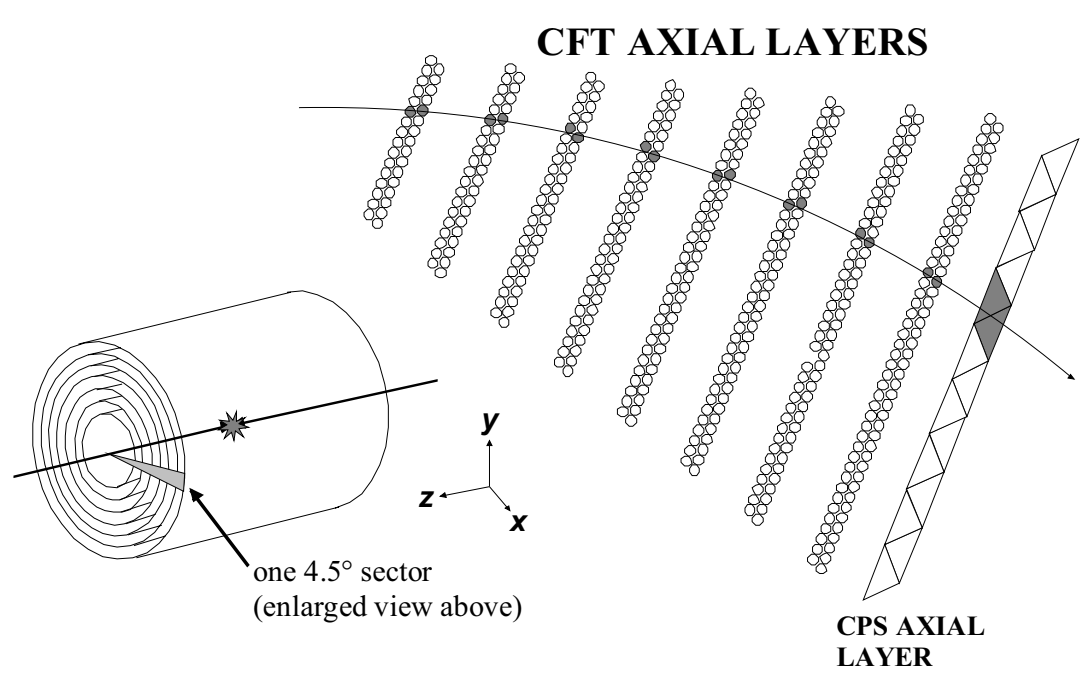

Figure 3.5: Transverse view of a $4.5^{\circ}$ trigger sector. A predefined track is overlaid on its associated hits in the CFT and CPS axial layers.

\begin{tabular}{|c|c|}
\hline $\operatorname{TTK}(\mathrm{n}, \mathrm{p})$ & n CFT track $(\mathrm{s})$ with $p_{T}>\mathrm{p}(\mathrm{p}=1.5,3,5,10 \mathrm{GeV})$ \\
\hline $\operatorname{TIS}(\mathrm{n}, \mathrm{p})$ & $\mathrm{n}$ isolated tracks with $p_{T}>\mathrm{p}(\mathrm{p}=5,10 \mathrm{GeV})$ \\
\hline THT(occ) & $\begin{array}{l}\text { Average fractional occupancy (fraction of CFT doublet } \\
\text { hits) in all sectors greater than occ }(\text { occ }=5,10,20 \%)\end{array}$ \\
\hline $\operatorname{TEL}(1, p)$ & CFT track with $p_{T}>\mathrm{p}$ with preshower deposition \\
\hline TIL & Isolated track with low home-sector occupancy \\
\hline $\operatorname{TPQ}(\mathrm{n}, \mathrm{p}, \mathrm{q})$ & $\begin{array}{l}\mathrm{n} \text { low } p_{T}(\mathrm{p}=1.5 \mathrm{GeV}) \mathrm{CFT} \text { track }(\mathrm{s}) \text { with preshower } \\
\text { deposition in quadrant } \mathrm{q}\end{array}$ \\
\hline $\operatorname{TNQ}(\mathrm{n}, \mathrm{q})$ & $\mathrm{n}$ preshower cluster(s) in quadrant q \\
\hline $\mathrm{TDL}(\mathrm{n}, \mathrm{p}, \mathrm{s})$ & $\begin{array}{l}\text { Pair of tracks with } p_{T}>\mathrm{p} \text {, with preshower match, with } \\
\text { same, opposite, or don't care charge signs }\end{array}$ \\
\hline $\operatorname{TDS}(\mathrm{n}, \mathrm{p}, \mathrm{s})$ & $\begin{array}{l}\text { Two isolated tracks }\left(p_{T}>5 \mathrm{GeV}\right) \text { with same or opposite } \\
\text { charge sign }\end{array}$ \\
\hline TAC(nsep) & $\begin{array}{l}\text { Track acoplanarity: number of sectors between two } \\
\text { highest } p_{T} \text { octants }>\text { nsep }\end{array}$ \\
\hline $\operatorname{TIQ}(\mathrm{n}, \mathrm{p}, \mathrm{q})$ & Isolated track in a quadrant \\
\hline $\operatorname{TOC}(n, p)$ & $\mathrm{n}$ octants with sum $p_{T}$ above $\mathrm{p}$ \\
\hline
\end{tabular}

Table 3.1: Level 1 CTT trigger terms. 
and isolated tracks above a certain $p_{T}$ threshold) are being used so far. The TIL and THT terms are in test phase. For the latter an additional difficulty comes from the measurement of luminosity [56]. The basic assumption is that there is at least one interaction (but no upper limit) for any bunch crossing that the luminosity monitor counts as live for DØ. Selecting events at L1 on a CFT occupancy less than some value would introduce a bias in this assumption by choosing preferentially crossings with a small number of interactions.

Each trigger may be live or disabled during different time intervals. To reduce the number of scalars needed to keep track of each particular L1 trigger dead time, they are grouped together in exposure groups: all triggers in a particular exposure group have common dead time, i.e. common sources of disable, enable and readout. All triggers of a same exposure group will therefore have a common correlation to the bunch structure.

Adding an occupancy term to an exposure group would cause the whole group to trigger preferentially on single interaction crossings, hence invalidating the basic assumption of luminosity measurement for all triggers, which would become unnormalisable. For that reason THT trigger terms are put in a separate exposure group. To be able to normalise them, it would be possible to put a zero-bias trigger (which triggers on each live bunch crossing) in this exposure group. Comparing the zero-bias rates in the main exposure group (where it is renormalisable) and in the THT group would give an idea of the luminosity in the THT group. But a precise measurement is difficult since the bunches triggered on are different (and not just a subset).

This is the reason why it was decided not to use such a trigger term (though desirable) for $W^{ \pm} \rightarrow \tau \nu_{\tau}$ events. Those events should have one or three tracks coming from the $\tau$ decay. The $p_{T}$ spectrum of such events is shown in Figure 3.6. Although $\tau$ leptons are produced with a $p_{T}$ around $40 \mathrm{GeV}$, the $p_{T}$ spectrum of tracks on which to trigger (coming from pions) is much lower. This hampers the ability to trigger efficiently on those events.

If one tries to trigger on a single track (either isolated or not), the background rejection decreases when the instantaneous luminosity increases: the activity in the detector increases, and it becomes more probable to get high $p_{T}$ tracks from QCD jets. A way to counter this effect is to select events where the track activity is low, i.e. with low occupancy. Figure 3.7 shows the occupancy distributions for $W^{ \pm} \rightarrow \tau \nu_{\tau}$ Monte Carlo signal events and for real data runs taken at two different luminosities. One can see the low occupancy in the signal case, while the background occupancy increases with luminosity: a cut selecting only low occupancy events would have given more rejection with increasing luminosity, counterbalancing the diminished rejection of the single track term.

The Level 1 trigger conditions for $W^{ \pm} \rightarrow \tau \nu_{\tau}$ events require a jet above $7 \mathrm{GeV}$ in the calorimeter and a track, either a TTK $(1,5)$ or a TIS $(1,10)$ term. Asking for a track above $5 \mathrm{GeV}$ is not sufficient to get a low enough rate 


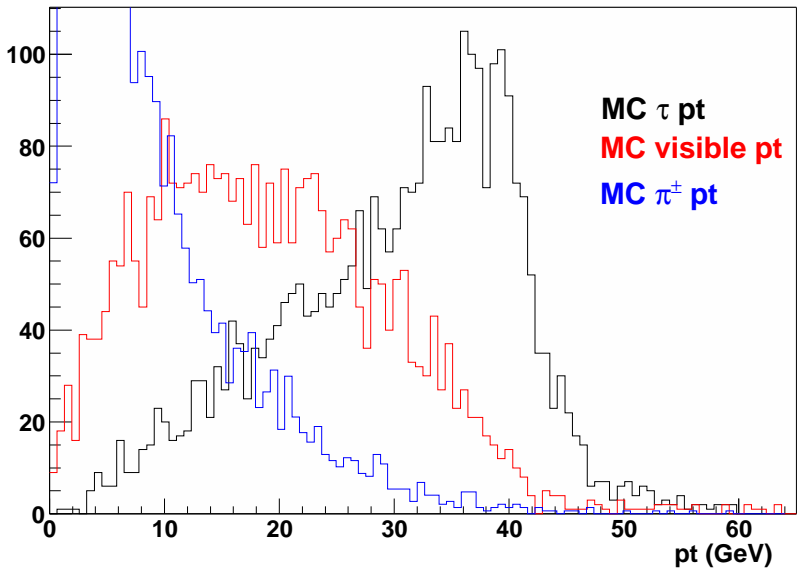

Figure 3.6: Transverse momentum of taus in $W^{ \pm} \rightarrow \tau \nu_{\tau}$ decays. The black curve is the $\tau p_{T}$, the red curve shows the visible tau $p_{T}$ and the blue curve represents the $p_{T}$ of charged particles (mostly pions) coming from $\tau$ decays. Those are the tracks on which one can trigger those events.

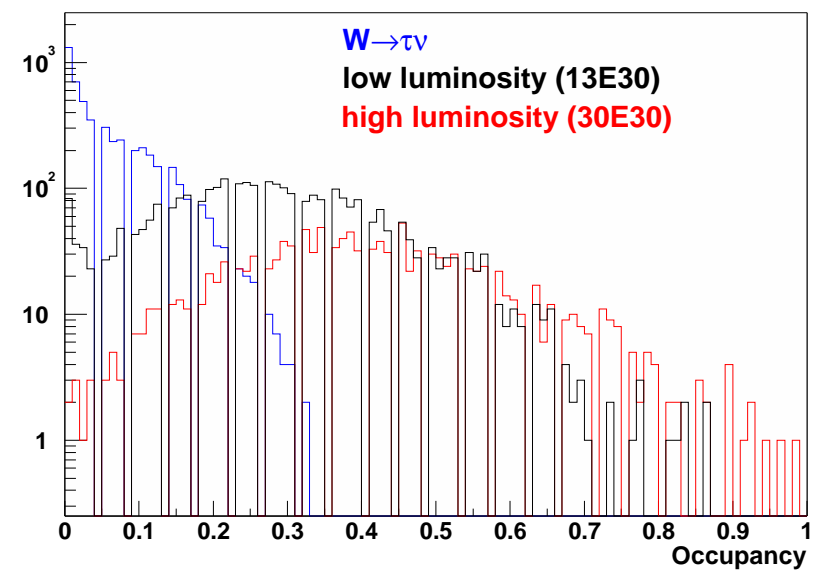

Figure 3.7: L1CTT occupancy for a $W^{ \pm} \rightarrow \tau \nu_{\tau}$ signal (blue curve) and for background taken at two different luminosities $\left(13 \cdot 10^{30} \mathrm{~cm}^{-2} \mathrm{~s}^{-1}\right.$ in black, $30 \cdot 10^{30} \mathrm{~cm}^{-2} \mathrm{~s}^{-1}$ in red). Note the logarithmic scale. 
at high luminosities, so this trigger is slightly prescaled. On the other hand an isolated $10 \mathrm{GeV}$ track gives enough rejection so that the L1 condition CJT $(1,7)$ TIS $(1,10)$ is unprescaled at all luminosities. Both triggers have been running online since 18 July 2003.

\subsubsection{Level 2 trigger}

The Level 2 trigger (L2) will run, according to the programming done in the trigger list, every time a L1 accept was issued, in order to bring the rate down by a factor of ten to $1 \mathrm{kHz}$ within $100 \mu \mathrm{s}$ [4]. It consists of two stages: preprocessors (described briefly in the following sections) specific to each subdetector prepare data from each L1 trigger subsystem, and a global processor (L2Global) combines objects from the preprocessors to make a trigger decision. This solution was preferred to the farm approach (where multiple events are processed in parallel) because of the requirement that trigger decisions be taken in event arrival order [57]. Figure 3.2 shows the relations between the different subsystems.

The L2 trigger is the first event-wide trigger decision: information from different subdetectors is combined into physics objects like muons, electrons or jets. L2Global makes quality requirements on preprocessor inputs, matches objects and calculates kinematic variables from multiple objects like angular separation or invariant masses [58].

There is a one-to-one mapping between L1 and L2 trigger bits. Each L1 specific trigger that fired will either be confirmed or rejected, resulting in a set of L2 trigger bits. This information is sent to the trigger framework and upon L2 accept (at least one L2 bit is satisfied) it tells Level 3 to initialise the detector precision readout for further trigger selection.

\section{Calorimeter preprocessor}

The calorimeter preprocessor system (L2CAL) [59] is running several clustering workers on the L1 trigger towers to identify EM objects (electrons and photons) and jets and compute missing transverse energy.

L1 trigger towers are turned into a seed mask: each tower with EM or total $E_{T}$ greater than a certain threshold (1 and $2 \mathrm{GeV}$ respectively) becomes a seed for clustering algorithms. The jet algorithm clusters together $3 \times 3$ or $5 \times 5$ groups of towers around a seed and sums up the total energy found in the 9 or 25 towers. All jets passing a minimum $E_{T}$ cut (set in the trigger list) are reported to L2Global together with the jet location. The EM clustering worker determines the highest $E_{T}$ neighbouring tower (among the four nearest) and adds its EM energy to that of the seed. In the trigger list one can cut on the EM $E_{T}$ of the object or on its EM fraction (the ratio of EM to total $E_{T}$ in the object). The missing transverse energy algorithm computes the vector sum of all trigger towers $E_{T}$ within a certain acceptance and reports it to L2Global. 


\section{Muon preprocessor}

The Level 2 muon system is more complex than the others, due to the large number of inputs and amount of processing required [60. Eighty digital signal processing units (DSPs) geographically organised in so-called SLICs (second level input computers) take the L1 muon outputs and incorporate calibration information and more precise timing from the scintillators to produce track segments in all subdetector regions. The SLIC results are sent to the muon preprocessor which matches track segments in the A and $\mathrm{BC}$ layers to create muon candidates and report them to L2Global with their associated $p_{T}$, location and quality.

\section{Tracking preprocessors}

The Level 2 central track trigger is composed of three preprocessors: CFT tracks are dealt with by L2CFT [61, L2STT 62] uses hits in the silicon detector (SMT) and L2PS 63] formats the output of the preshower detectors (CPS and FPS). None of these systems are fully commissioned to this day.

The L2CFT receives tracks from L1CTT, formats them for L2 (i.e. transforms $\eta$ and $p_{T}$ bins into physical quantities) and sorts them in $p_{T}$ before sending them to L2Global. The preshower preprocessor L2PS formats L1CTT preshower clusters and sends them to L2Global where they can be correlated to L2CFT tracks and to L2CAL electron candidates, hence increasing the ability to trigger on electrons.

The L2STT was the last feature added to the D $\varnothing$ trigger system in 1999 64. It will take input from L1CTT and will perform precise reconstruction of tracks using data from the SMT in addition. Around each L1 CFT track a $\pm 1 \mathrm{~mm}$ road is defined and SMT clusters found within the road are considered for the fit. When L2STT will be available online it will also serve as an input to L2CTT which will combine the track fit parameters with L1 CFT tracks and transmit the results to L2Global. At this stage one can select events with large impact parameter tracks indicating the presence of long-lived particles such as $b$ quarks.

\subsubsection{Level 3 trigger and data acquisition}

If an event satisfies the L1 and L2 conditions of a specific trigger, a L2 accept is issued and the event is processed by the Level 3 trigger (L3), the last trigger step. The precision readout is now available and data is handled from the detector crates to the storage unit by the data acquisition system (DAQ) described in the next section. The L3 trigger system uses the precision readout to reduce the rate of events stored on tape for further physics analysis. 


\section{Data acquisition architecture}

The $\mathrm{D} \varnothing$ data acquisition system was completely and successfully redesigned in 2002 to an Ethernet-based framework [65]. The DAQ should handle simultaneous runs (i.e. sets of L1/L2/L3 and detector conditions): anytime a new run is started, the Supervisor will make sure that all needed resources and configurations are available and properly loaded, so that data taking is possible. It will notify the farm nodes on which L3 processing will be done (see next section) about the software and trigger parameters they should use and tell the Routing Master (RM) about the new run.

The RM program runs on a single board computer (SBC). It synchronises data transfer between the subdetectors and the farm nodes. When a L2 accept is issued the RM will decide, depending on the fired L2 bit, which subdetectors should be read out and to which node to send the data. SBCs on each detector crate called by the RM read the data and send it to a farm node for L3 processing. The RM also sends to the node the relevant information about the incoming data: event number, fired L1/L2 triggers and crate list, so that the node can check whether it has received all necessary data before starting processing. In the case when the last node ready to receive data is allocated, the RM will apply back-pressure on the L1 trigger system by asking the trigger framework to disable the associated triggers until a node can accept a new event.

Each farm node can then start to process the data. First the event builder (EVB) combines the event fragments received from the different SBCs to form complete events, according to the crate list specification sent by the RM. Once the event is complete it is distributed to the different L3 processes on the machine. The event is reconstructed and a trigger decision is taken. If it passes it is sent to the Collector which will record it for further offline analysis, otherwise it is discarded. Details of the trigger decision process are given in the next section.

\section{Level 3 filtering}

The nominal data handling requirement for the Level 3 trigger system is to bring down the $1 \mathrm{kHz}$ input rate from Level 2 to a maximum of $50 \mathrm{~Hz}$ within $\sim 100 \mathrm{~ms}$, each event saved on tape being approximately $250 \mathrm{kB} 66$. The data input, event building and output formatting taking around $15 \mathrm{~ms}$ this leaves about $85 \mathrm{~ms}$ per event for unpacking, reconstruction and filtering. It is performed on a Linux farm with 48 dual processor nodes, straightforward to expand if necessary.

The L3 filtering is controlled by SCRIPTRunneR [67] which determines the trigger decision on each event. At the beginning of a run it parses the trigger list to make a map of all available tools and filters. For every fired L2 bit it causes one or more L3 filter scripts associated to this L2 bit to be run. A filter script consists of the logical AND of one or more filters. If any filter script returns success, the event is flagged to be written out. 
There are three types of filters. Trigger filters handle specific trigger list requirements like prescale factors or Mark\&Pass fractions (for debugging purposes it can be decided to record a certain fraction of events, the Mark\&Pass fraction, whatever the outcome of the filter scripts). Physics object filters require the presence of one or more physics objects satisfying a set of criteria. Relational filters use the output of one or more physics filters to compute composite objects like invariant mass or object separation.

The objects used by filters are produced by L3 tools called by the filters. Tools can also provide their results to each other. Several tools of the same flavour (for instance several tau tools) can coexist, each having a specific set of configuration parameters and a unique name. There are four kinds of tools. Trigger tools take care of basic functionalities used by trigger filters like error handling. Unpacking tools access raw data, apply calibration and alignment corrections and unpack the regions of interest of the detector requested by the data tools. Those data tools perform partial reconstruction of the event (in order to save processing time) like calorimeter clustering or track reconstruction. They provide their results to filters or to other higher level tools, the physics tools, in charge of running the different algorithms to find physics object candidates. The physics tools are the tools steering the whole process: for instance the tau tool asks for calorimeter clusters from the clustering tool which in turn needs the calorimeter unpacker tool input.

Since the same tool may be called several times (for instance the calorimeter clustering tool may be called by several instances of the jet and tau tools), tools are required by design to cache their results in order to save CPU time. It is only once the whole trigger list has been processed that SCRIPTRUnNER will tell the tools to reinitialise themselves in preparation for the next event.

A certain number of physics tools are already used for data taking: calorimeter jets, electrons, muons (either in the muon chambers or with a central track or calorimeter match), tracks, primary vertex and missing transverse energy. More are expected to be used soon like a CPS tool, secondary vertex and $b$-jet tagging.

For this thesis a particular tool, the tau tool, was studied and brought online to trigger on $Z \rightarrow \tau \tau$ events. This work is reported in Paper VI. It can serve as an example for how Level 3 works. Consider a trigger designed to select $Z \rightarrow \tau(e) \tau($ had) events. At Level 3 it requires an electron and a tau objects. For efficiency studies one would also like to have a sample of events that failed the selection. To perform all those requirements a filter script could contain three filters: first a Mark\&Pass filter with a pass fraction 1 of 200; then an electron filter asking for an electron candidate with $E_{T}>10 \mathrm{GeV}$; finally a tau filter requiring a tau candidate above $10 \mathrm{GeV}$. The electron and tau candidates will be produced by instances of the electron and tau tools defined by a set of parameters (like the minimum profile of the tau candidates or the shower shape of the electrons). The electron and 
tau tools will make use of the clustering data tool which will itself call the calorimeter unpacker tool and the vertex tool. All the different parameters and configurations described above are programmed in the trigger list.

Now this filter script is associated to a L2 bit. Every time this bit fires, SCRIPTRUnNer calls each filter of the script one after the other. The Mark\&Pass filter will either return success or flag the event as unbiased one out of fifty times. If there is an electron candidate above $10 \mathrm{GeV}$ the electron filter is passed and the next filter is called. Otherwise the filter fails and SCRIPTRunNer flags the event as failed. If the event is unbiased, the next filter is called, otherwise it exits the filter script: there is no point in processing further since the event won't be recorded anyway. In case the next filter was called, the tau filter will check for a tau candidate above $10 \mathrm{GeV}$. In the end the filter script status is passed if all filters passed and failed otherwise. In addition it returns whether the event was unbiased. If the script passed or is unbiased the event is recorded, otherwise it is discarded.

As for each new tool, the tau tool had to go through a certification procedure, using the trigger simulator D0TRIGSIM which has an offline interface to SCRIPTRUnNER. The filtering code has to be reliable: there is no second chance for reprocessing if an event was wrongly discarded online. It should also be robust to keep execution errors at a minimum. Although development at $\mathrm{D} \varnothing$ is done on different platforms, the L3 code must be fully tested under Linux as it is the operating system on the farm. It has to work "out-of-the-box" to minimise human intervention and allow traceability of the code run online. It has to run without crashing on large samples of real data and Monte Carlo events, both when run alone and together with other tools. Memory consumption must be under control and timing studies must be performed.

The final stage is the formal approval by the Trigger Board after the tool performances have been demonstrated. It can then be included in a trigger list and run online. The Level 3 tau tool has been running online since 17 January 2002. 


\section{Chapter 4}

\section{Summary of papers}

\section{Paper I}

The reconstruction of the $\tau-\tau$ invariant mass from observed decay products was studied in ATLAS. Fully simulated $Z$ +jets events were generated for different ' $Z$ masses'. This study assumed no knowledge of the missing transverse energy, to which not only the $\tau$ neutrinos may contribute (for instance in some supersymmetric final states). It was shown that using hadronic $\tau$ decays, one can select taus efficiently enough (for supersymmetric analyses) using calorimeter isolation and jet calorimeter mass.

Combining information from the tracking system and the calorimeter, the $\tau$-jet mass was reconstructed. Cutting on this variable helped selecting $\tau$ decays where the visible decay products carry a large fraction of the $\tau$ energy. The $\tau-\tau$ invariant mass was reconstructed, giving a better resolution than would be the case if all hadronic taus were selected. The results were parameterised for easy use in fast simulation.

\section{Paper II}

An estimation of the statistical and systematic uncertainties in the measurement of the CP-violation parameter $\sin 2 \beta$ in ATLAS is presented. The $B_{d}^{0} \rightarrow J / \psi K_{s}^{0}$ decay channel was fully reconstructed, different tagging algorithms were tested and background rejection was measured to estimate the statistical uncertainty on $\sin 2 \beta$.

The systematic uncertainties were studied using the $B_{d}^{0} \rightarrow J / \psi K_{s}^{* 0}$ and $B^{+} \rightarrow J / \psi K^{+}$control channels. They should exhibit negligible CPviolating effects and are very similar to the signal, so their reconstruction (in large numbers) allows the measurement of the uncertainty on the $B / \bar{B}$ production asymmetry and on the wrong tag fraction.

The estimated statistical and systematic errors in the measurement of $\sin 2 \beta$, after three years of running at low luminosity $\left(30 \mathrm{fb}^{-1}\right)$ are 0.010 
and 0.005 , respectively, for an assumed value of $\sin 2 \beta$ of 0.60 .

\section{Paper III}

The hadronic $\tau$ decay of a heavy charged Higgs boson, $H^{ \pm} \rightarrow \tau \nu_{\tau}$, was studied with the ATLAS detector. Only the $H^{ \pm}$mass region above the top quark mass was considered, when the charged Higgs is produced via the $g b \rightarrow t H^{ \pm}$process. The dominant $H^{ \pm}$decay mode is then $H^{ \pm} \rightarrow t b$, which suffers from large irreducible backgrounds.

But for high values of $\tan \beta$, a non negligible fraction of $H^{ \pm}$decays to $\tau \nu_{\tau}$. Using the kinematic differences of the signal and backgrounds, the transverse mass can be reconstructed in an almost background-free environment. The different polarisation state of taus coming from $W^{ \pm}$and $H^{ \pm}$ decays enhances the signal and reduces the background.

This study led to a better coverage of the $\left(m_{A}, \tan \beta\right)$ parameter space at the $5 \sigma$ discovery level for $\tan \beta>10$ above the top quark mass and up to $\sim 600 \mathrm{GeV}$.

\section{Paper IV}

The results obtained in Paper III served as a basis to measure the uncertainty on the determination of the charged Higgs boson mass and of $\tan \beta$.

A maximum likelihood method was used to obtain the statistical mass uncertainty. Systematic effects like the background shape and rate and the energy scale were also included. It was shown that the total uncertainty, of the order of 1 to $2 \%$ over the $H^{ \pm}$mass range $200-500 \mathrm{GeV}$ for an integrated luminosity of $300 \mathrm{fb}^{-1}$, is dominated by the statistical errors.

The measurement of the absolute rate of $H^{ \pm} \rightarrow \tau \nu_{\tau}$ allows the computation of the uncertainty on the determination of $\tan \beta$. The main systematic error is on the luminosity measurement, and leads to an overall precision on $\tan \beta$ around $6 \%$ for a charged Higgs mass of $250 \mathrm{GeV}$ and $\tan \beta$ between 20 and 50 , with $300 \mathrm{fb}^{-1}$ of integrated luminosity.

\section{Paper V}

The sensitivity of the ATLAS detector to the discovery of a heavy charged Higgs boson is presented in this article which includes all available ATLAS results on the subject. Assuming a heavy SUSY mass spectrum, the most promising channels above the top quark mass are $H^{ \pm} \rightarrow t b$ and $H^{ \pm} \rightarrow \tau \nu_{\tau}$ which provide coverage in the low and high $\tan \beta$ regions up to $\sim 600 \mathrm{GeV}$. The achievable precisions on the charged Higgs mass and $\tan \beta$ determination are also presented.

The reconstruction of the channel $H^{-} \rightarrow \tau_{L}^{-} \psi$ is also discussed. It may provide a distinctive evidence for models with singlet neutrinos in large 
extra dimension scenarios by measuring the polarisation asymmetry. The fraction of the energy carried by the charged track in the one-prong $\tau$ decay would help to pin down the actual scenario, 2HDM or not.

\section{Paper VI}

The tau tool for the DØ Level 3 trigger is described. Technical details of its implementation and use are given. Two different algorithms can be used to make $\tau$ objects at Level 3. One can either search for calorimeter clusters with some shape properties and then check for tracks pointing towards them, or first make track clusters and gather calorimeter clusters around them.

Performance on $Z \rightarrow \tau \tau$ events, QCD and data was studied. Efficiency and rejection are reported, as well as the impact of different levels of online calorimeter zero suppression. Timing of the tool, a crucial variable when running online, was also studied. This tau tool has been running online in DØ since 17 January 2002. 



\section{Chapter 5}

\section{Conclusions and outlook}

The work presented in this thesis is part of the effort to find experimental evidence for deviations from the Standard Model predictions. Observing such divergences would demonstrate the need for a more general theory than the Standard Model, like, e.g. Supersymmetry.

By measuring precisely the unitarity triangle parameter $\sin 2 \beta$ the SM description of CP violation can be tested. This requires control of the systematic uncertainties so that they do not spoil the precision that can be achieved at very high statistics. The Monte Carlo study performed for ATLAS shows that the systematic uncertainty is half the attainable statistical uncertainty. The total expected uncertainty is almost an order of magnitude smaller than the most recent results reported from $B$ factories at $e^{+} e^{-}$ colliders [13, supporting the case of a $B$ physics program for the ATLAS experiment.

The tau lepton's distinctive signature is a useful tool in many new physics searches where it is present in the final state. It was studied at the ATLAS experiment to parameterise the detector response to two-tau final states. Such a scenario is common, in particular in Supersymmetry. Measuring the end point of the $\tau-\tau$ invariant mass distribution could provide information about the underlying structure of supersymmetric models.

Several extensions of the SM predict the existence of a charged Higgs boson. The major part of this thesis has consisted in using tau leptons to search for a charged Higgs in the context of the Minimal Supersymmetric extension of the SM. Results from this thesis show that the $H^{ \pm} \rightarrow \tau \nu_{\tau}$ decay channel extends the $H^{ \pm}$discovery reach for the ATLAS experiment compared to previous studies of other channels: the charged Higgs can be observed for masses up to $\sim 600 \mathrm{GeV}$ for $\tan \beta>10$. Its mass can be determined with an uncertainty of 1 to $2 \%$, dominated by statistical errors. The $\tan \beta$ parameter can be derived from the absolute rate of the $H^{ \pm} \rightarrow \tau \nu_{\tau}$ decay to a precision around $6 \%$ for $20<\tan \beta<50$.

A lot of work was performed at the $\mathrm{D} \emptyset$ experiment to develop the ability 
to trigger on events containing taus. This is the first necessary step in preparation for the carrying out of a search for the charged Higgs at D $\varnothing$. It also contributes to future new physics searches at the $\mathrm{D} \varnothing$ experiment.

The charged Higgs boson has raised a lot of interest in the past few years. The possibility of observing a particle that has no equivalent in the Standard Model is one main reason: observing it would definitely prove that there must be a broader theoretical framework than the SM.

Several studies have recently been performed that claim to enhance the potential for charged Higgs discovery at the Tevatron and the LHC experiments 68 or to broaden the scope for charged Higgs searches by looking at pair production [69] or supersymmetric decays [70]. But several uncertainties remain as to what the $H^{ \pm}$production cross section actually is and theoretical work is underway to get better estimates [71.

A future linear collider will offer excellent opportunities to look for and study the charged Higgs. This has already been studied in different collision and decay modes 72 . The next generation linear colliders, beyond the LHC era, with a project like CLIC (Compact Linear Collider) at CERN, will continue to contribute to charged Higgs physics. The reach in mass and precision would be significantly increased [73.

The prospects for charged Higgs discovery at $\mathrm{D} \varnothing$ are naturally more limited but Run 2 should extend significantly the reach as compared to Run 1 74. It has been frustrating for me to see how slowly the experiment is converging towards a fully commissioned detector and high quality physics results. When the Uppsala group started discussing the possibility to join a Fermilab experiment, the aim was to perform a charged Higgs search with the new $\mathrm{D} \varnothing$ detector. A few years later, the observation of the comparatively much higher cross section process $W^{ \pm} \rightarrow \tau \nu_{\tau}$ is still around the corner for $\mathrm{D} \varnothing$, so that only work at the $\tau$ trigger level has been possible to achieve for this thesis. Nevertheless the detector and its trigger system are persistently getting better and the Tevatron will be the highest energy running collider for the coming five years, with concomitant opportunities to make unexpected discoveries. 


\section{Acknowledgements}

Producing a doctoral thesis is a rather long process. Over the years I spent to carry through this project, many people have contributed to my work and to my wellbeing. This page is for them all.

I would like to thank my supervisor Tord Ekelöf for believing in me from the beginning and for giving me the opportunity to participate in two related but very different projects: it made me a better physicist.

Many thanks go to Kétévi Assamagan for a very fruitful collaboration and for being such a good friend every time we met at CERN. Thanks also to Jérôme Damet for sharing the French office and spirit in Uppsala and introducing me to the refinements of ATLAS and $B$ physics.

I am indebted to Nils Gollub, Richard Brenner, Mattias Ellert and Christophe Clément for proofreading parts of my manuscript, and to Inger Ericson for her relentless help and support, especially while I was staying at Fermilab.

During the year and a half I spent at Fermilab I met many people. I would particularly like to thank the $\mathrm{D} \varnothing$ Sweden group and the volleyball and football (or soccer as they call it over there) players for taking me out of my office and making my stay enjoyable. My thanks go also to all my friends and colleagues at Fermilab and Uppsala.

I thank warmly my parents and family for always being there for me, whatever decision I made.

My most profound feelings go to you, Reilika. You do not have to be jealous of my thesis anymore: my close relationship with it is over! Thank you for everything we have done together all those years and for the years to come: ma armastan sind. 



\section{References}

[1] B. R. Martin and G. Shaw, Particle Physics, Chichester: John Wiley \& Sons, 1992.

[2] P. Aliani, V. Antonelli, M. Picariello, and E. Torrente-Lujan, Neutrino mass parameters from Kamland, SNO and other solar evidence, (2003), hep-ph/0212212.

[3] M. K. Gaillard, P. D. Grannis, and F. J. Sciulli, The standard model of particle physics, Rev. Mod. Phys. 71 (1999) S96, hep-ph/9812285.

[4] C. L. Bennett et al., First year Wilkinson Microwave Anisotropy Probe (WMAP) observations: preliminary maps and basic results, (2003), astro-ph/0302207.

[5] G. 't Hooft and M. J. G. Veltman, Regularization and renormalization of gauge fields, Nucl. Phys. B44 (1972) 189.

[6] P. W. Higgs, Broken symmetries, massless particles and gauge fields, Phys. Lett. 12 (1964) 132; P. W. Higgs, Broken symmetries and the masses of gauge bosons, Phys. Rev. Lett. 13 (1964) 508; F. Englert and R. Brout, Broken symmetry and the mass of gauge vector mesons, Phys. Rev. Lett. 13 (1964) 321.

[7] S. L. Glashow, Partial symmetries of weak interactions, Nucl. Phys. 22 (1961) 579; S. Weinberg, A model of leptons, Phys. Rev. Lett. 19 (1967) 1264; A. Salam, Weak and electromagnetic interactions, in 8th Nobel Symposium, ed. N. Svartholm, Stockholm: Almqvist \& Wiksell, 1968.

[8] J. F. Gunion, H. E. Haber, G. L. Kane, and S. Dawson, The Higgs Hunter's Guide, Perseus Publishing, 2000.

[9] ALEPH Collaboration, DELPHI Collaboration, L3 Collaboration, OPAL Collaboration and The LEP Working Group for Higgs Boson Searches, Search for the standard model Higgs boson at LEP, Phys. Lett. B565 (2003) 61, hep-ex/0306033. 
[10] N. Cabibbo, Unitary symmetry and leptonic decays, Phys. Rev. Lett. 10 (1963) 531; M. Kobayashi and T. Maskawa, CP violation in the renormalizable theory of weak interaction, Prog. Theor. Phys. 49 (1973) 652 .

[11] L. Wolfenstein, Parametrization of the Kobayashi-Maskawa matrix, Phys. Rev. Lett. 51 (1983) 1945.

[12] J. H. Christenson, J. W. Cronin, V. L. Fitch, and R. Turlay, Evidence for the $2 \pi$ decay of the $K_{2}^{0}$ meson, Phys. Rev. Lett. 13 (1964) 138.

[13] The BABAR Collaboration, Measurement of the CP-violating asymmetry amplitude $\sin 2 \beta$, Phys. Rev. Lett. 89 (2002) 201802, hepex/0207042 The Belle Collaboration, An improved measurement of mixing-induced $\mathrm{CP}$ violation in the neutral B meson system, Phys. Rev. D66 (2002) 071102, hep-ex/0208025.

[14] K. Hagiwara et al., Review of particle physics, Phys. Rev. D66 (2002).

[15] F. Matorras, Tau physics at LEP, eConf C0209101 (2002) TU02, hep-ex/0211054, invited talk at 7th International Workshop on Tau Lepton Physics (TAU 02), Santa Cruz, California, 10-13 Sep 2002.

[16] J. R. Ellis, Supersymmetry for Alp hikers, (2002), hep-ph/0203114.

[17] S. L. Glashow and S. Weinberg, Natural conservation laws for neutral currents, Phys. Rev. D15 (1977) 1958.

[18] ALEPH, DELPHI, L3 and OPAL Collaborations, CERN-EP/2000055; ALEPH Collaboration, CERN-EP/2000-086, Phys. Lett. B 487 (2000) 253; DELPHI Collaboration, CERN-EP/2001-062, M. Ellert et al., Nucl. Phys. B - Proceeding supplements 98 (2001), 336; L3 Collaboration, CERN-EP/2000-118, Phys. Lett. B 496 (2000) 34; OPAL Collaboration, CERN-EP/98-173.

[19] P. Gutierrez, FERMILAB-Conf-00-294-E; V. M. Abazov et al. [DØ Collaboration], Direct search for charged higgs bosons in decays of top quarks, Phys. Rev. Lett. 88 (2002) 151803, hep-ex/0102039.

[20] A. Krause, T. Plehn, M. Spira, and P. M. Zerwas, Production of charged Higgs boson pairs in gluon gluon collisions, Nucl. Phys. B519 (1998) 85, hep-ph/9707430; Y. Jiang, W.-g. Ma, L. Han, M. Han, and Z.-h. Yu, SUSY charged Higgs boson pair production via gluongluon collisions, J. Phys. G24 (1998) 83, hep-ph/9708421; A. A. Barrientos Bendezu and B. A. Kniehl, $\mathrm{H}^{+} \mathrm{H}^{-}$pair production at the Large Hadron Collider, Nucl. Phys. B568 (2000) 305, hep-ph/9908385; O. Brein and W. Hollik, Pair production of charged MSSM Higgs bosons by gluon fusion, Eur. Phys. J. C13 (2000) 175, hep-ph/9908529. 
[21] D. A. Dicus, J. L. Hewett, C. Kao, and T. G. Rizzo, $W^{ \pm} H^{\mp}$ production at hadron colliders, Phys. Rev. D40 (1989) 787; A. A. Barrientos Bendezu and B. A. Kniehl, $W^{ \pm} H^{\mp}$ associated production at the Large Hadron Collider, Phys. Rev. D59 (1999) 015009, hep-ph/9807480 Quark-loop amplitudes for $W^{ \pm} H^{\mp}$ associated hadroproduction, Phys. Rev. D61 (2000) 097701, hep-ph/9909502 Squark loop correction to $W^{ \pm} H^{\mp}$ associated hadroproduction, Phys. Rev. D63 (2001) 015009, hep-ph/0007336; O. Brein, W. Hollik, and S. Kanemura, The MSSM prediction for $W^{ \pm} H^{\mp}$ production by gluon fusion, Phys. Rev. D63 (2001) 095001, hep-ph/0008308.

[22] S. Moretti and K. Odagiri, The phenomenology of $W^{ \pm} H^{\mp}$ production at the Large Hadron Collider, Phys. Rev. D59 (1999) 055008, hep$\mathrm{ph} / 9809244$.

[23] F. Borzumati, J.-L. Kneur, and N. Polonsky, Charged-Higgs-strahlung and R-parity violating slepton-strahlung at hadron colliders, Phys. Rev. D60 (1999) 115011, hep-ph/9905443 S. Moretti and D. P. Roy, Detecting heavy charged Higgs bosons at the LHC with triple btagging, Phys. Lett. B470 (1999) 209, hep-ph/9909435 A. Djouadi et al., The Higgs working group: Summary report, (2000), hepph/0002258, D. Denegri et al., Summary of the CMS discovery potential for the MSSM SUSY Higgses, (2001), hep-ph/0112045; A. Belyaev, D. Garcia, J. Guasch, and J. Sola, Prospects for supersymmetric charged Higgs boson discovery at the Tevatron and the LHC, Phys. Rev. D65 (2002) 031701, hep-ph/0105053.

[24] K. A. Assamagan, The charged Higgs in hadronic decays with the ATLAS detector, Acta Phys. Polon. B31 (2000) 863; Signature of the charged Higgs decay $H^{ \pm} \rightarrow W h^{0}$ with the ATLAS detector, Acta Phys. Polon. B31 (2000) 881.

[25] D. Cavalli et al., Search for $H^{ \pm} \rightarrow \tau \nu_{\tau}$ decays, ATLAS Note ATLPHYS-94-053 (1994); C. Biscarat, Search for charged Higgs bosons produced in top quark decays with the ATLAS detector at the LHC, ATLAS Note ATL-SLIDE-2003-002.

[26] LHC yellow book, CERN-AC-95-05 LHC (1995), most recent updates at http://lhc-new-homepage.web.cern.ch.

[27] The ATLAS Collaboration, ATLAS technical proposal for a generalpurpose pp experiment at the Large Hadron Collider at CERN, CERNLHCC-94-43 (1994).

[28] The ATLAS Collaboration, ATLAS inner detector technical design report, CERN/LHCC/97-16 (1997).

[29] The ATLAS Collaboration, Liquid argon technical design report, CERN/LHCC 96-41 (1996). 
[30] The ATLAS Collaboration, Tile calorimeter technical design report, CERN/LHCC 96-42 (1996).

[31] T. Sjöstrand, High-energy physics event generation with PYTHIA 5.7 and JETSET 7.4, Comput. Phys. Commun. 82 (1994) 74.

[32] GEANT detector description and simulation tool, CERN Program Library, W5013 (1994).

[33] I. Gavrilenko, Description of global pattern recognition program (XKalman), ATL-INDET-97-165 (1997).

[34] E. Richter-Wass, D. Froidevaux, and L. Poggioli, ATLFAST 2.0 a fast simulation package for ATLAS, ATLAS Note ATL-PHYS-98-131 (1998).

[35] The D $\varnothing$ Collaboration: S. Abachi et al, Observation of the top quark, Phys. Rev. Lett. 74 (1995) 2632, hep-ex/9503003.

[36] The DØ Collaboration, D $\varnothing$ silicon tracker technical design report, D $\varnothing$ Note 2169 (1994), http://d0server1.fnal.gov/projects/silicon/www/tdr_final.ps.

[37] The D $\varnothing$ Collaboration, The D $\varnothing$ upgrade central fiber tracker technical design report, (1997), http://d0server1.fnal.gov/users/stefan/www/CFT_TDR/CFT_TDR.ps

[38] The D $\varnothing$ Collaboration, Design report of the central preshower detector for the DØ upgrade, DØ Note 3014 (1996).

[39] The DØ Collaboration, Technical design report of the forward preshower detector for the DØ upgrade, DØ Note 3445 (1998).

[40] The DØ Collaboration: S. Abachi et al, The DØ detector, Nucl. Instrum. Meth. A338 (1994) 185.

[41] The DØ Collaboration, Calorimeter electronics upgrade for Run 2, (1998), http://www-d0.fnal.gov/ d0upgrad/calelec/intro/tdr/tdr17.pdf

[42] The D $\varnothing$ Collaboration, Technical design report for the upgrade of the ICD for DØ Run II, DØ Note 2686 (1997).

[43] The DØ Collaboration, Technical design of the central muon system, DØ Note 3365 (1997).

[44] The DØ Collaboration, Technical design report for the $\mathrm{D} \varnothing$ forward muon tracking detector based on mini-drift tubes, D $\varnothing$ Note 3366 (1997). 
[45] A.Brandt et al., A forward proton detector at D $\varnothing$, FERMILAB-Pub$97 / 377$ (1997).

[46] A. Lo, C. Miao, and R. Partridge, Luminosity monitor technical design report, DØ Note 3320 (1997).

[47] G. C. Blazey, The DØ Run II trigger, in Proceedings of 10th IEEE RealTime Computer Applications in Nuclear, Particle and Plasma Physics (RT 97), Beaune, France, 1997, FERMILAB-CONF-97-395-E.

[48] http://www-d0.fnal.gov/computing/trigsim/trigsim.html.

[49] M. Abolins, D. Edmunds, S. Gross, and P. Laurens, D $\varnothing$ Run II Level 1 trigger framework technical design report, (1998), http://www.pa.msu.edu/hep/d0/ftp/l1/framework/l1fw_tdr_05june98.txt.

[50] M. Abolins, D. Edmunds, P. Laurens, J. Linnemann, and B. Pi, The Level One calorimeter trigger for DØ, DØ Note 706 (1988), http://www.pa.msu.edu/hep/d0/ftp/run1/l1/caltrig/d0_note_706.txt.

[51] M. Klute and A. Quadt, Measurements of Level 1 trigger efficiencies from DØ data, DØ Note 3949 (2002).

[52] D. Chakraborty, Y. Coadou, S. Duensing, C. Galea, Y. Gershtein, AC. LeBihan, C. Noeding and S. Protopopescu, Reconstruction of tau leptons in hadronic final states at D $\varnothing$ in Run 2, DØ Note 4210 (2003).

[53] J. Butler et al., Level 1 muon trigger technical design report, (1999), http://atlas.physics.arizona.edu/ johns/l1muo/l1mu/Summary.PDF.

[54] CTT group, Technical design report for the upgrade L1/L2 tracking trigger - TDR for the CTT, DØ Note 3949 (1999).

[55] L. Babukhadia and M. Martin, Track and preshower digital trigger in DØ, DØ Note 3980 (2002).

[56] M. Begel, private communication.

[57] R. D. Martin, Design of the D $\varnothing$ Run II Level 2 trigger, International Conference on Computing in High-Energy Physics (CHEP 98), Chicago, Illinois (1998),

http://hepalpha1.phy.uic.edu/l2cal/misc/chep133.ps;

R. Hirosky, The D-Zero Level 2 trigger for Run II physics, 11th IEEE NPSS Real Time Conference (RT99), Santa Fe, New Mexico (1999), http://www-d0.fnal.gov/ hirosky/talks/RT133.ps.

[58] D. Edmunds, S. Gross, P. Laurens, J. Linnemann, and R. Moore, Technical design report for the Level 2 global processor, DØ Note 3402 (1998),

http://www.pa.msu.edu/hep/d0/ftp/l2/overview/globaltdr/global_tdr.pdf. 
[59] M. Adams et al., Level-2 calorimeter preprocessor technical design report, DØ Note 3651 (1999), http://hepalpha1.phy.uic.edu/12cal/12cal_tdr_v1_5.ps.

[60] C. Leonidopoulos, The muon trigger at DØ, DØ Note 4099 (2003), Computing In High-Energy Physics And Nuclear (CHEP'01), Beijing, China.

[61] D. Toback, A. Baden, F. Borcherding, and K. Yip, Specification of the Level 2 central tracking trigger preprocessor crate, DØ Note 3645 (2002), http://www.nhn.ou.edu/〜abbott/L2Trigger/12ctt_documentation_02_5.ps.

[62] The DØ Collaboration, A silicon track trigger for the D $\varnothing$ experiment in Run II, DØ Note 3516 (1998); M. Narain, A silicon track trigger for the DØ experiment, Nucl. Instrum. Meth. A447 (2000) 223.

[63] M. Bhattacharjee and P. Grannis, Technical design report: Level 2 preprocessor for central and forward preshower triggers, (1999), http://www-d0.fnal.gov/ mrinmoy/L2_TDR.ps.gz.

[64] G. Steinbrück, The DØ silicon track trigger, (2002), 11th International Workshop On Vertex Detectors (Vertex 2002), Kona, Kailua, Hawaii, http://www-d0.fnal.gov/trigger/stt/papers/gsVertex2002_proc.pdf.

[65] B. Angstadt et al., Ethernet-based data acquisition for the D $\varnothing$ experiment at Fermilab, in Proceedings of the 6th World Multiconference on Systemics, Cybernetics and Informatics (SCI2002), Orlando, Florida, 2002 .

[66] A. Boehnlein, G. Brooijmans, D. Claes, and M. Souza, Description of the DØ L3 trigger software components, DØ Note 3630 (1999).

[67] M. Souza, ScriptRunner implementation notes, (1999), http://wwwd0.fnal.gov/ moacyr/l3/impl.ps.

[68] S. Moretti, Improving the discovery potential of charged Higgs bosons at the Tevatron and Large Hadron Collider, Pramana 60 (2003) 369, hep-ph/0205104.

[69] S. Moretti and J. Rathsman, Pair production of charged Higgs bosons in association with bottom quark pairs at the Large Hadron Collider, (2003), hep-ph/0308215.

[70] M. Bisset, F. Moortgat, and S. Moretti, Trilepton + top signal from chargino neutralino decays of MSSM charged Higgs bosons at the LHC, (2003), hep-ph/0303093. 
[71] A. Belyaev, J. Guasch, and J. Sola, Supersymmetric effects on heavy charged Higgs boson production in hadron colliders, Nucl. Phys. Proc. Suppl. 116 (2003) 296, hep-ph/0210253; T. Plehn, Charged Higgs boson production in bottom gluon fusion, Phys. Rev. D67 (2003) 014018, hep-ph/0206121; Charged Higgs boson production in bottom-gluon fusion, prepared for 10th International Conference on Supersymmetry and Unification of Fundamental Interactions (SUSY02), Hamburg, Germany, 17-23 Jun 2002; Les Houches workshop 2003: physics at TeV colliders, http://wwwlapp.in2p3.fr/conferences/LesHouches/Houches2003/index.html

[72] S. Moretti and S. Kanemura, Heavy charged Higgs boson production at next generation $\gamma \gamma$ colliders, Eur. Phys. J. C29 (2003) 19, hepph/0211055 S. Moretti, Detection of heavy charged Higgs bosons in $e^{+} e^{-} \rightarrow t b H^{-}$production at future linear colliders, (2003), hepph/0306297; Detection of heavy charged Higgs bosons at future linear colliders via $\tau^{-} \bar{\nu}_{\tau} H^{+}$production, (2002), hep-ph/0209210; Detection of heavy charged Higgs bosons at future linear colliders, Eur. Phys. J. direct C4 (2002) 15, hep-ph/0206208.

[73] A. Ferrari, Pair production of charged Higgs bosons in $e^{+} e^{-}$collisions at CLIC, LC Note LC-PHSM-2003-051 (2003).

[74] D. Chakraborty, Charged Higgs bosons, in Report of the Tevatron Higgs working group, edited by M. Carena, J. S. Conway, H. E. Haber, and J. D. Hobbs, 2000, hep-ph/0010338. 



\title{
Appendix A
}

\section{Abbreviations and acronyms}

\author{
2HDM Two Higgs Doublet Model \\ ALICE A Large Ion Collider Experiment (LHC experiment) \\ AMSB Anomaly Mediated SUSY Breaking \\ ATLAS A Toroidal LHC Apparatus (LHC experiment) \\ BLS BaseLine Subtractor \\ $\mathrm{CC} \quad$ Central Calorimeter (D $\varnothing$ subdetector) \\ CDF Collider Detector at Fermilab (Tevatron experiment) \\ CERN European Laboratory for Particle Physics (accelerator labo- \\ ratory near Geneva, Switzerland) \\ CFT Central Fiber Tracker (DØ subdetector) \\ CKM Cabibbo-Kobayashi-Maskawa \\ CMS Compact Muon Solenoid (LHC experiment) \\ CPS Central PreShower detector (DØ subdetector) \\ CPU Central Processing Unit \\ DØ DZero (Tevatron experiment) \\ DAQ Data AcQuisition system \\ DSP Digital Signal Processing \\ EC End Calorimeter (DØ subdetector) \\ EM Electromagnetic; electromagnetic calorimeter (D $\varnothing$ subdetec- \\ tor) \\ $E_{T} \quad$ Transverse energy \\ $\mathbb{E}_{T} \quad$ Missing transverse energy \\ EVB EVent Builder (DØ subsystem) \\ FCNC Flavour Changing Neutral Current \\ FNAL Fermi National Accelerator Laboratory, aka Fermilab (accel- \\ erator laboratory near Chicago, Illinois, USA) \\ FPD Forward Proton Detector (DØ subdetector)
}


FPS Forward PreShower detector (DØ subdetector)

GMSB Gauge Mediated SUSY Breaking

GUT Grand Unified Theory

ICD Inter Cryostat Detector (D $\varnothing$ subdetector)

L $n \quad$ Level $n$ trigger at $\mathrm{D} \varnothing(n=1,2,3)$

L1CAL Level 1 CALorimeter trigger (D $\varnothing$ subsystem)

L1CTT Level 1 Central Track Trigger (DØ subsystem)

L1FW Level 1 Trigger Framework (DØ subsystem)

L1Muo Level 1 Muon trigger (DØ subsystem)

L2CAL Level 2 CALorimeter preprocessor (D $\varnothing$ subsystem)

L2CFT Level 2 Central Fiber Tracker preprocessor (DØ subsystem)

L2Global Level 2 Global preprocessor (DØ subsystem)

L2PS Level 2 PreShower preprocessor (DØ subsystem)

L2STT Level 2 Silicon Track Trigger preprocessor (DØ subsystem)

LEP Large Electron Positron Collider (accelerator at CERN, closed in 2000)

LHC Large Hadron Collider (future accelerator at CERN, due 2007)

$\mathrm{LHCb}$ Large Hadron Collider beauty experiment (LHC experiment)

LINAC LINear ACcelerator

LM Luminosity Monitor (DØ subdetector)

MDT Mini-Drift Tube

MSSM Minimal Supersymmetric extension of the Standard Model

PDT Proportional Drift Tube

PS Proton Synchrotron (accelerator at CERN)

$p_{T} \quad$ Transverse momentum

QCD Quantum Chromodynamics

QED Quantum Electrodynamics

RM Routing Master (DØ subsystem)

SCT SemiConductor Tracker (ATLAS subdetector)

SBC Single Board Computer

SLIC Second Level Input Computer (D $\varnothing$ subsystem)

SM Standard Model of particle physics

SMT Silicon Microstrip Tracker (DØ subdetector)

SPS Super Proton Synchrotron (accelerator at CERN)

SUGRA SUperGRAvity

SUSY SUperSYmmetry

TRT Transition Radiation Tracker (ATLAS subdetector)

vev Vacuum expectation value

VLPC Visible Light Photon Counter 


\section{Acta Universitatis Upsaliensis}

\section{Comprehensive Summaries of Uppsala Dissertations}

from the Faculty of Science and Technology

Editor: The Dean of the Faculty of Science and Technology

A doctoral dissertation from the Faculty of Science and Technology, Uppsala University, is usually a summary of a number of papers. A few copies of the complete dissertation are kept at major Swedish research libraries, while the summary alone is distributed internationally through the series Comprehensive Summaries of Uppsala Dissertations from the Faculty of Science and Technology. (Prior to October, 1993, the series was published under the title "Comprehensive Summaries of Uppsala Dissertations from the Faculty of Science".)

Distribution:

Uppsala University Library

Box 510, SE-751 20 Uppsala, Sweden

www.uu.se, acta@ub.uu.se 